\title{
HYBRID GAUSS-TRAPEZOIDAL QUADRATURE RULES
}

Bradley K. Alpert

QC 



\section{HYBRID GAUSS-TRAPEZOIDAL QUADRATURE RULES}

\section{Bradley K. Alpert}

Applied and Computational Mathematics Division Computing and Applied Mathematics Laboratory National Institute of Standards and Technology Boulder, Colorado 80303-3328

July 1996

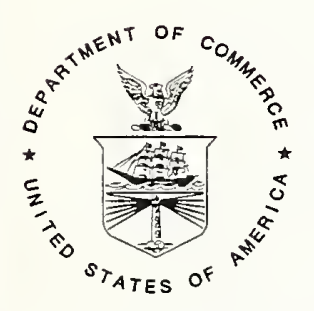




\title{
HYBRID GAUSS-TRAPEZOIDAL QUADRATURE RULES
}

\author{
BRADLEY K. ALPERT \\ Applied and Computational Mathematics Division \\ National Institute of Standards and Technology, Boulder, Colorado 80303
}

\begin{abstract}
A new class of quadrature rules for the integration of both regular and singular functions is constructed and analyzed. For each rule the quadrature weights are positive and the class includes rules of arbitrarily high-order convergence. The quadratures result from alterations to the trapezoidal rule, in which a small number of nodes and weights at the ends of the integration interval are replaced. The new nodes and weights are determined so that the asymptotic expansion of the resulting rule, provided by a generalization of the Euler-Maclaurin summation formula, has a prescribed number of vanishing terms. The superior performance of the rules is demonstrated with numerical examples and application to several problems is discussed.
\end{abstract}

KEY WORDS. Euler-Maclaurin formula, Gaussian quadrature, high-order convergence, numerical integration, positive weights, singularity

\section{CONTENTS}

1. Introduction

2. Mathematical Preliminaries 3

2.1. Bernoulli Polynomials 3

2.2. Euler-Maclaurin Summation Formula 4

2.3. Generalized Riemann Zeta-Function 4

2.4. Orthogonal Polynomials and Gaussian Quadrature 5

3. Hybrid Gauss-Trapezoidal Quadrature Rules 6

3.1. Regular Integrands 6

3.2. Singular Integrands 8

3.3. Improper Integrals 11

4. Existence and Uniqueness 13

4.1. Chebyshev Systems 13

4.2. Müntz System Quadratures 15

5. Computation of the Nodes and Weights 18

5.1. Regular Integrands 18

5.2. Singular Integrands $\quad 19$

6. Numerical Examples 21

6.1. Nodes and Weights 22

6.2. Quadrature Performance 23

7. Applications and Summary 25

Acknowledgments $\quad 27$

$\begin{array}{ll}\text { References } & 27\end{array}$

Appendix. Tables of Quadrature Nodes and Weights 29

E-mail address: alpert@boulder.nist.gov. 


\section{INTRODUCTION}

The well-known Euler-Maclaurin summation formula provides an asymptotic expansion for the trapezoidal rule applied to regular functions. While the constant term of the expansion is an integral, the other terms depend on the integrand's derivatives at the endpoints of the interval of integration. This expansion is often used to "correct" the trapezoidal rule to a quadrature with high-order convergence, through the use of either known derivative values or their finite-difference approximations.

In this paper we derive asymptotic expansions, analogous to the Euler-Maclaurin formula, for functions with known singularities. In particular, functions with power or logarithmic singularities are treated. The Euler-Maclaurin formula and the new asymptotic expansions are used to construct quadrature rules of arbitrary order convergence. Each quadrature is constructed by altering the trapezoidal rule: a few of the nodes and weights at the interval endpoints are replaced with new nodes and weights determined so as to annihilate several terms in the asymptotic expansion. The nodes always lie within the interval of integration and the weights are always positive.

For a regular function $f:[0,1] \rightarrow \mathbb{R}$, we approximate $\int_{0}^{1} f(x) d x$ with the quadrature

$$
\begin{aligned}
\tilde{T}_{n}(f)=h\left[w_{1} f\left(x_{1} h\right)+w_{2}\right. & f\left(x_{2} h\right)+\cdots+w_{j} \\
& f\left(x_{j} h\right) \\
& +f(a h)+f(a h+h)+\cdots+f(1-a h) \\
& \left.+w_{j} f\left(1-x_{j} h\right)+\cdots+w_{1} f\left(1-x_{1} h\right)\right] .
\end{aligned}
$$

There are $n$ "internal" nodes with spacing $h=1 /(n+2 a-1)$ and $j$ "endpoint" nodes at each end, with the endpoint nodes $x_{1}, \ldots, x_{j}$ and weights $w_{1}, \ldots, w_{j}$ chosen so that the asymptotic expansion of $\tilde{T}_{n}$ as $n \rightarrow \infty$ has $2 j$ vanishing terms and

$$
\tilde{T}_{n}(f)=\int_{0}^{1} f(x) d x+O\left(h^{2 j+1}\right)
$$

(Theorem 3.1 and Corollary 3.2). The parameters $a$ and $j$, and the nodes $x_{1}, \ldots, x_{j}$ and weights $w_{1}, \ldots, w_{j}$, are independent of $n$. The nodes and weights are determined by $2 j$ nonlinear equations, which have a unique solution, with

$$
0<x_{i}<a, \quad w_{i}>0, \quad i=1, \ldots, j,
$$

provided $a$ is sufficiently large (Theorem 4.7 ). For integrands that are singular at one endpoint, $\tilde{T}_{n}$ is altered so that the nodes and weights at that end differ from those at the other end and depend on the singularity (Theorem 3.4 and Corollary 3.6; Theorem3.7 and Corollary 3.8). For improper integrals in which the integrand is oscillatory and slowly decaying, $\tilde{T}_{n}$ is combined with Gauss-Laguerre quadrature to give rules with high-order convergence (Theorem 3.9 and Corollary 3.10).

Several authors have studied the problems treated here. It has been observed that endpoint corrections can be derived for singular integrands; Rokhlin [1] implemented such a scheme for integrands with a known singularity at an interval endpoint. He derived corrections to the trapezoidal rule by placing additional quadrature nodes near the endpoint, with the corresponding weights determined so that low-order polynomials and the singularity times low-order polynomials were integrated exactly. He showed that under fairly general conditions, these weights had limiting values (up to scale) as the number of nodes in the trapezoidal rule increased without bound and that these limiting weights could be used to form quadrature rules with good convergence. Unfortunately, the order of convergence of these rules is restricted in practice by the fact that the weights increase in magnitude rapidly 
as the order increases. Efforts by Starr [2] and subsequently Alpert [3] reduced the growth in size of the weights with order, primarily by using more weights than the number of equations satisfied and minimizing their sum of squares. In another approach, Kress [4] uses all quadrature weights in the quadrature rule, rather than a few near the endpoints, to handle the singularity. More recently, Kapur and Rokhlin [5] successfully constructed rules of arbitrary order by separating the integrand's regular and singular parts and allowing some quadrature nodes to lie outside the interval of integration.

The present approach does not suffer from limitations on order of convergence, separation of the integrand into parts, or quadrature nodes outside the interval of convergence. On the other hand, the quadrature nodes near the interval endpoints are not equispaced. Also, the equations for the nodes $x_{1}, \ldots, x_{j}$ and weights $w_{1}, \ldots, w_{j}$, in addition to being nonlinear, are poorly conditioned; the conditioning deteriorates rapidly with increasing order. Nevertheless, we are able to use an algorithm developed recently by Ma, Rokhlin, and Wandzura [6] for computing generalized Gaussian quadratures to obtain accurate quadrature nodes and weights. The author would also like to credit that paper for inspiring the present work.

The paper is organized around Section 3, where the new asymptotic expansions are derived and the quadratures defined, and Section 4 , where it is shown that the equations defining the quadratures actually have solutions, which are unique. These sections are preceded by mathematical preliminaries and followed by a discussion of the computation of the quadrature nodes and weights. Numerical examples are presented in Section 6 and we conclude with some applications and a summary.

\section{Mathematical Preliminaries}

The material in this section, which is found in standard references, is used in the subsequent development.

2.1. Bernoulli Polynomials. The Bernoulli polynomials are defined by generating function (see, for example, Abramowitz and Stegun [7] 23.1.1),

$$
\frac{t e^{x t}}{e^{t}-1}=\sum_{n=0}^{\infty} B_{n}(x) \frac{t^{n}}{n !}
$$

from which

$$
B_{0}(x)=1, \quad B_{1}(x)=x-\frac{1}{2}, \quad B_{2}(x)=x^{2}-x+\frac{1}{6}, \quad B_{3}(x)=x^{3}-\frac{3}{2} x^{2}+\frac{1}{2} x .
$$

The Bernoulli polynomials satisfy the difference formula

$$
\frac{B_{n}(x+k)-B_{n}(x)}{n}=\sum_{i=0}^{k-1}(x+i)^{n-1}, \quad n=1,2, \ldots,
$$

the differentiation formula

$$
B_{n}^{\prime}(x)=n B_{n-1}(x), \quad n=1,2, \ldots
$$

and the expansion formula

$$
B_{n}(x+h)=\sum_{r=0}^{n}\left(\begin{array}{l}
n \\
r
\end{array}\right) B_{r}(x) h^{n-r}, \quad n=0,1, \ldots
$$


2.2. Euler-Maclaurin Summation Formula. For a function $f \in C^{p}(\mathbb{R})$ with $p \geq 1$, the EulerMaclaurin summation formula (see, for example, Abramowitz and Stegun [7] 23.1.30) can be derived by repeated integration by parts. We first consider the interval $[c, c+h]$ and apply (5) to obtain

$$
\begin{aligned}
\int_{c}^{c+h} f(x) d x= & h \int_{0}^{1} B_{0}(1-x) f(c+x h) d x \\
= & -\left.h \frac{B_{1}(1-x)}{1 !} f(c+x h)\right|_{0} ^{1}+h^{2} \int_{0}^{1} \frac{B_{1}(1-x)}{1 !} f^{\prime}(c+x h) d x \\
& \vdots \\
= & -\left.\sum_{r=0}^{p-1} \frac{h^{r+1} B_{r+1}(1-x)}{(r+1) !} f^{(r)}(c+x h)\right|_{0} ^{1} \\
& +h^{p+1} \int_{0}^{1} \frac{B_{p}(1-x)}{p !} f^{(p)}(c+x h) d x \\
= & h \frac{f(c)+f(c+h)}{2}-\sum_{r=1}^{p-1} \frac{h^{r+1} B_{r+1}}{(r+1) !}\left[f^{(r)}(c+h)-f^{(r)}(c)\right] \\
& +h^{p+1} \int_{0}^{1} \frac{B_{p}(1-x)}{p !} f^{(p)}(c+x h) d x,
\end{aligned}
$$

where we have used

$$
\begin{aligned}
-B_{1}(0) & =B_{1}(1)=\frac{1}{2} \\
B_{n}(0) & =B_{n}(1)=B_{n}, \quad n \neq 1 .
\end{aligned}
$$

To derive the Euler-Maclaurin formula for the interval $[a, b]$, we let $h=(b-a) / n$ and $c=a+i h$ in (7), sum over $i=0,1, \ldots, n-1$, and rearrange terms, to obtain

$$
\begin{aligned}
h\left[\frac{f(a)}{2}+f(a+h)\right. & \left.+\cdots+f(b-h)+\frac{f(b)}{2}\right] \\
=\int_{a}^{b} f(x) d x+ & \sum_{r=1}^{p-1} \frac{h^{r+1} B_{r+1}}{(r+1) !}\left[f^{(r)}(b)-f^{(r)}(a)\right] \\
& -h^{p+1} \int_{0}^{1} \frac{B_{p}(1-x)}{p !}\left\{\sum_{i=0}^{n-1} f^{(p)}(a+i h+x h)\right\} d x .
\end{aligned}
$$

The expression on the left-hand side of (8) is the well-known trapezoidal rule. Evaluation of the expression on the right-hand side of (8) is simplified by the fact that $B_{2 r+1}=0$ for $r \geq 1$.

2.3. Generalized Riemann Zeta-Function. The generalized Riemann $\zeta$-function is defined by the formula

$$
\zeta(s, v)=\sum_{n=0}^{\infty} \frac{1}{(v+n)^{s}}, \quad \operatorname{Re}(s)>1, v \neq 0,-1, \ldots .
$$

This function has a continuation that is analytic in the entire complex $s$-plane, with the exception of $s=1$, where it has a simple pole. In what follows, we shall be concerned primarily with real $s$ and 
$v$, with $s<1$ and $v>0$. We will use the following representation derived from Plana's summation formula (see, for example, Erdélyi [8] 1.10 (7)):

$$
\zeta(s, v)=\frac{v^{1-s}}{s-1}+\frac{v^{-s}}{2}+2 v^{1-s} \int_{0}^{\infty} \frac{\sin (s \arctan t)}{\left(1+t^{2}\right)^{s / 2}} \frac{d t}{e^{2 \pi v t}-1}, \quad \operatorname{Re}(v)>0 .
$$

Equation (9) can be used to derive the asymptotic expansion of $\zeta$ as $v \rightarrow \infty$. We treat the integral as a sum of Laplace integrals, each with an asymptotic expansion given by Watson's lemma (see, for example, Bender and Orszag [9] p. 263), and obtain

$$
\zeta(s, v)=\frac{v^{1-s}}{s-1}+\frac{v^{-s}}{2}+\frac{1}{s-1} \sum_{r=1}^{p}\left(\begin{array}{c}
s+2 r-2 \\
2 r
\end{array}\right) \frac{B_{2 r}}{v^{s+2 r-1}}+O\left(v^{-s-2 p-1}\right),
$$

as $v \rightarrow \infty$, with $s \in \mathbb{C}, s \neq 1$, and $p$ an arbitrary positive integer. Equation (10) is a slight generalization of [8] 1.18 (9). There is a direct connection between the Bernoulli polynomials and $\zeta$,

$$
\frac{B_{n}(x)}{n}=-\zeta(1-n, x), \quad n=1,2, \ldots,
$$

and generalizations of the difference and differentiation formulae hold:

$$
\begin{gathered}
\zeta(s, v)-\zeta(s, v+k)=\sum_{i=0}^{k-1} \frac{1}{(v+i)^{s}}, \\
\frac{\partial \zeta(s, v)}{\partial v}=-s \zeta(s+1, v) .
\end{gathered}
$$

2.4. Orthogonal Polynomials and Gaussian Quadrature. Suppose that $\omega$ is a positive continuous function on the interval $(a, b)$ and $\omega$ is integrable on $[a, b]$. We define the inner product with respect to $\omega$ of real-valued functions $f$ and $g$ by the integral

$$
(f, g)=\int_{a}^{b} f(x) g(x) \omega(x) d x .
$$

There exist polynomials $p_{0}, p_{1}, p_{2}, \ldots$, of degree $0,1,2, \ldots$, respectively, such that $\left(p_{n}, p_{m}\right)=0$ for $n \neq m$ (orthogonality); they are unique up to the choice of leading coefficients. With leading coefficients one, they can be obtained recursively by the formulae (see, for example, Stoer and Bulirsch [10], p. 143)

$$
\begin{aligned}
p_{0}(x) & =1 \\
p_{n+1}(x) & =\left(x-\delta_{n+1}\right) p_{n}(x)-\gamma_{n+1}^{2} p_{n-1}(x), \quad n \geq 0,
\end{aligned}
$$

where $p_{-1}(x)=0$ and $\delta_{n}, \gamma_{n}$ are defined by the formulae

$$
\begin{aligned}
& \delta_{n+1}=\left(x p_{n}, p_{n}\right) /\left(p_{n}, p_{n}\right), \quad n \geq 0, \\
& \gamma_{n+1}{ }^{2}= \begin{cases}0 & n=0 \\
\left(p_{n}, p_{n}\right) /\left(p_{n-1}, p_{n-1}\right) & n \geq 1 .\end{cases}
\end{aligned}
$$

The zeros $x_{1}^{n}, \ldots, x_{n}^{n}$ of $p_{n}$ are distinct and lie in the interval $(a, b)$. There exist positive numbers $\omega_{1}^{n}, \ldots, \omega_{n}^{n}$ such that

$$
\int_{a}^{b} f(x) \omega(x) d x=\sum_{i=1}^{n} \omega_{i}^{n} f\left(x_{i}^{n}\right)
$$


whenever $f$ is a polynomial of degree less than $2 n$. These Christoffel numbers are given by the formula (see, for example, Szegö [11] p. 48)

$$
\omega_{i}^{n}=\frac{\left(p_{n-1}, p_{n-1}\right)}{p_{n-1}\left(x_{i}^{n}\right) p_{n}^{\prime}\left(x_{i}^{n}\right)}, \quad i=1, \ldots, n .
$$

Moreover, if $\omega(x)=(b-x) \tau(x)$ with $\tau$ integrable on $[a, b]$, then, with the definition $x_{n+1}^{n}=b$, there exist positive numbers $\tau_{1}^{n}, \ldots, \tau_{n+1}^{n}$ such that

$$
\int_{a}^{b} f(x) \tau(x) d x=\sum_{i=1}^{n+1} \tau_{i}^{n} f\left(x_{i}^{n}\right)
$$

whenever $f$ is a polynomial of degree less than or equal to $2 n$. These modified Christoffel numbers are given by the formula

$$
\tau_{i}^{n}= \begin{cases}\frac{\omega_{i}^{n}}{b-x_{i}^{n}} & i=1, \ldots, n, \\ \int_{a}^{b} \frac{p_{n}(x)}{p_{n}(b)} \tau(x) d x & i=n+1,\end{cases}
$$

where $\omega_{i}^{n}$ is given by (16).

The summation in (15) is the $n$ node Gaussian quadrature with respect to $\omega$, while that in (17) is an $n+1$ node Gauss-Radau quadrature with respect to $\tau$.

\section{Hybrid Gauss-Trapezoidal Quadrature Rules}

In this section we introduce new quadrature rules for regular integrands, singular integrands with a power or logarithmic singularity, and improper integrals, and determine their rate of convergence as the number of quadrature nodes increases.

For notational convenience we generally consider quadratures on canonical intervals, primarily $[0,1]$. It is understood that these are readily transformed to quadratures on any finite interval $[a, b]$ by the appropriate linear transformation of the nodes and weights.

3.1. Regular Integrands. For $j, n$ positive integers and $a \in \mathbb{R}^{+}=\{x \in \mathbb{R} \mid x>0\}$, we define a linear operator $T_{n}^{j a}$ on $C([0,1])$, depending on nodes $x_{1}, \ldots, x_{j}$ and weights $w_{1}, \ldots, w_{j}$, by the formula

$$
T_{n}^{j a}(f)=h \sum_{i=1}^{j} w_{i} f\left(x_{i} h\right)+h \sum_{i=0}^{n-1} f(a h+i h)+h \sum_{i=1}^{j} w_{i} f\left(1-x_{i} h\right),
$$

where $h=(n+2 a-1)^{-1}$ is chosen so that $a h+(n-1) h=1-a h$.

Theorem 3.1. Suppose $f \in C^{p}([0,1])$. The asymptotic expansion of $T_{n}^{j a}(f)$ as $n \rightarrow \infty$ is given by the formula

$$
\begin{aligned}
& T_{n}^{j a}(f)=\int_{0}^{1} f(x) d x \\
& \quad+\sum_{r=0}^{p-1} h^{r+1} \frac{f^{(r)}(0)+(-1)^{r} f^{(r)}(1)}{r !}\left\{\sum_{i=1}^{j} w_{i} x_{i}^{r}-\frac{B_{r+1}(a)}{r+1}\right\}+O\left(h^{p+1}\right) .
\end{aligned}
$$


Proof. We apply the Euler-Maciaurin formula (8) on the interval $[a h, 1-a h]$ to obtain

$$
\begin{aligned}
& h \sum_{i=0}^{n-1} f(a h+i h)=h \frac{f(a h)+f(1-a h)}{2}+\int_{a h}^{1-a h} f(x) d x \\
& \quad+\sum_{r=1}^{p-1} \frac{h^{r+1} B_{r+1}}{(r+1) !}\left[f^{(r)}(1-a h)-f^{(r)}(a h)\right]+O\left(h^{p+1}\right) .
\end{aligned}
$$

We now combine (19) and (21), the equality

$$
\int_{a h}^{1-a h} f(x) d x=\int_{0}^{1} f(x) d x-\int_{0}^{a h} f(x) d x-\int_{1-a h}^{1} f(x) d x,
$$

Taylor expansion of all quantities about $h=0$, the Bernoulli polynomial expansion formula (6), and difference formula (4) to obtain (20).

Corollary 3.2. Suppose the nodes $x_{1}, \ldots, x_{j}$ and weights $w_{1}, \ldots, w_{j}$ satisfy the equations

$$
\sum_{i=1}^{j} w_{i} x_{i}^{r}=\frac{B_{r+1}(a)}{r+1}, \quad r=0,1, \ldots, 2 j-1 \text {. }
$$

Then $T_{n}^{j a}$ is a quadrature rule with convergence of order $2 j+1$ for $f \in C^{p}([0,1])$ with $p \geq 2 j$. Moreover,

$$
T_{n}^{j a}(f)-\int_{0}^{1} f(x) d x \sim h^{2 j+1} \frac{f^{(2 j)}(0)+f^{(2 j)}(1)}{(2 j) !}\left\{\sum_{i=1}^{j} w_{i} x_{i}^{2 j}-\frac{B_{2 j+1}(a)}{2 j+1}\right\}
$$

as $n \rightarrow \infty$, provided $f^{(2 j)}(0)+f^{(2 j)}(1) \neq 0$.

Corollary 3.3. Suppose $x_{j}=a-1$ and the remaining nodes $x_{1}, \ldots, x_{j-1}$ and weights $w_{1}, \ldots, w_{j}$ satisfy the equations

$$
\sum_{i=1}^{j} w_{i} x_{i}^{r}=\frac{B_{r+1}(a)}{r+1}, \quad r=0,1, \ldots, 2 j-2
$$

Then $T_{n}^{j a}$ is a quadrature rule with convergence of order $2 j$ for $f \in C^{p}([0,1])$ with $p \geq 2 j-1$. Moreover,

$$
T_{n}^{j a}(f)-\int_{0}^{1} f(x) d x \sim h^{2 j} \frac{f^{(2 j-1)}(0)-f^{(2 j-1)}(1)}{(2 j-1) !}\left\{\sum_{i=1}^{j} w_{i} x_{i}{ }^{2 j-1}-\frac{B_{2 j}(a)}{2 j}\right\}
$$

as $n \rightarrow \infty$, provided $f^{(2 j-1)}(0)-f^{(2 j-1)}(1) \neq 0$.

We shall see below that (22) has a solution with the nodes and weights all positive if $a$ is sufficiently large and that numerical solution of (22) is equivalent to computing the roots of a particular polynomial. This statement holds for (24) as well. 
3.2. Singular Integrands. For $j, k, n$ positive integers and $a, b \in \mathbb{R}^{+}$, we define a linear operator $S_{n}^{j k a b}$ on $C((0,1])$, depending on nodes $v_{1}, \ldots, v_{j}, x_{1}, \ldots, x_{k}$ and weights $u_{1}, \ldots, u_{j}, w_{1}, \ldots, w_{k}$, by the formula

$$
S_{n}^{j k a b}(g)=h \sum_{i=1}^{j} u_{i} g\left(v_{i} h\right)+h \sum_{i=0}^{n-1} g(a h+i h)+h \sum_{i=1}^{k} w_{i} g\left(1-x_{i} h\right),
$$

where $h=(n+a+b-1)^{-1}$ is chosen so that $a h+(n-1) h=1-b h$.

Theorem 3.4. Suppose $g(x)=x^{\gamma} f(x)$, where $\gamma>-1$ and $f \in C^{p}([0,1])$. The asymptotic expansion of $S_{n}^{j k a b}(g)$ as $n \rightarrow \infty$ is given by the formula

$$
\begin{aligned}
S_{n}^{j k a b}(g)=\int_{0}^{1} g(x) & d x+\sum_{r=0}^{p-1} h^{\gamma+r+1} \frac{f^{(r)}(0)}{r !}\left\{\sum_{i=1}^{j} u_{i} v_{i}^{\gamma+r}+\zeta(-\gamma-r, a)\right\} \\
& +\sum_{r=0}^{p-1} h^{r+1} \frac{(-1)^{r} g^{(r)}(1)}{r !}\left\{\sum_{i=1}^{k} w_{i} x_{i}^{r}-\frac{B_{r+1}(b)}{r+1}\right\}+O\left(h^{p+1+\min \{0, \gamma]}\right) .
\end{aligned}
$$

Proof. For $c \in \mathbb{R}^{+}$, we define polynomials $p_{0}^{c}, p_{1}^{c}, \ldots$, in analogy with the Bernoulli polynomials, by the formula

$$
p_{n}^{c}(x)=\sum_{r=0}^{n}\left(\begin{array}{l}
n \\
r
\end{array}\right) \zeta(-\gamma-r, c)(1-c-x)^{n-r} .
$$

Differentiating, we verify that

$$
\frac{d}{d x} p_{n}^{c}(x)=-n p_{n-1}^{c}(x), \quad n=1,2, \ldots
$$

and combining the $\zeta$ difference formula (11) with (28) we obtain

$$
p_{n}^{c}(1)-p_{n}^{c+1}(0)= \begin{cases}c^{\gamma} & n=0 \\ 0 & n \geq 1\end{cases}
$$

Additionally, we define functions $q_{0}^{c}, q_{1}^{c}, \ldots$ by the formula

$$
q_{n}^{c}(x)= \begin{cases}(x+c)^{\gamma} & n=0, \\ \frac{(-1)^{n}(x+c)^{\gamma+n}}{(\gamma+1) \cdots(\gamma+n)}-\frac{p_{n-1}^{c+1}(x)}{(n-1) !} & n=1,2, \ldots\end{cases}
$$

and observe that

$$
\frac{d}{d x} q_{n}^{c}(x)=-q_{n-1}^{c}(x), \quad n=1,2, \ldots
$$


With these definitions, the proof follows that of the Euler-Maclaurin formula.

$$
\begin{aligned}
\int_{a h}^{1-b h} x^{\gamma} f(x) d x= & \sum_{i=0}^{n-2} h^{1+\gamma} \int_{0}^{1} q_{0}^{a+i}(x) f(a h+i h+x h) d x \\
= & \sum_{i=0}^{n-2}\left\{-\left.\sum_{r=0}^{p-1} h^{\gamma+r+1} q_{r+1}^{a+i}(x) f^{(r)}(a h+i h+x h)\right|_{0} ^{1}\right. \\
& \left.\quad+h^{\gamma+p+1} \int_{0}^{1} q_{p}^{a+i}(x) f^{(p)}(a h+i h+x h) d x\right\} \\
= & h \sum_{i=1}^{n-2}(a h+i h)^{\gamma} f(a h+i h) \\
& -\sum_{r=0}^{p-1} h^{\gamma+r+1}\left[q_{r+1}^{a+n-2}(1) f^{(r)}(1-b h)-q_{r+1}^{a}(0) f^{(r)}(a h)\right] \\
& +h^{\gamma+p+1} \int_{0}^{1}\left\{\sum_{i=0}^{n-2} q_{p}^{a+i}(x) f^{(p)}(a h+i h+x h)\right\} d x .
\end{aligned}
$$

Taylor expansion of $f^{(r)}(a h)$ about $h=0$, the definitions (28) and (29) for $p_{n}^{c}$ and $q_{n}^{c}$, and the binomial theorem combine to yield

$$
\begin{aligned}
\sum_{r=0}^{p-1} h^{\gamma+r+1} q_{r+1}^{a}(0) & f^{(r)}(a h)= \\
& -\sum_{k=0}^{p-1} \frac{h^{\gamma+k+1} f^{(k)}(0)}{k !}\left[\frac{a^{\gamma+k+1}}{\gamma+k+1}+\zeta(-\gamma-k, a+1)\right]+O\left(h^{\gamma+p+1}\right) .
\end{aligned}
$$

Likewise, Taylor expansion of $f^{(r)}(1-b h)$ about $h=0$, the definitions for $p_{n}^{c}$ and $q_{n}^{c}$, the asymptotic expansion (10) for $\zeta$, the Bernoulli polynomial expansion formula (6), and the binomial theorem combine to yield

$$
\begin{aligned}
& \sum_{r=0}^{p-1} h^{\gamma+r+1} q_{r+1}^{a+n-2}(1) f^{(r)}(1-b h)= \\
& \sum_{k=0}^{p-1} \frac{h^{k+1}(-1)^{k} g^{(k)}(1)}{k !}\left[\frac{b^{k+1}}{k+1}-\frac{B_{k+1}(b+1)}{k+1}\right]+O\left(h^{p+1}\right) .
\end{aligned}
$$

We now combine (26) and (30) through (32), the equality

$$
\int_{a h}^{1-b h} g(x) d x=\int_{0}^{1} g(x) d x-\int_{0}^{a h} g(x) d x-\int_{1-b h}^{1} g(x) d x,
$$

expansion of the latter two integrals about $h=0$, and the difference formula (4) for the Bernoulli polynomials and (11) for $\zeta$ to obtain (27). 
Corollary 3.5. Suppose the nodes $u_{1}, \ldots, u_{j}$ and the weights $v_{1}, \ldots, v_{j}$ satisfy the equations

$$
\sum_{i=1}^{j} u_{i} v_{i}^{\gamma+r}=-\zeta(-\gamma-r, a), \quad r=0,1, \ldots, 2 j-1,
$$

and the nodes $x_{1}, \ldots, x_{j}$ and weights $w_{1}, \ldots, w_{j}$ satisfy the equations

$$
\sum_{i=1}^{j} w_{i} x_{i}^{r}=\frac{B_{r+1}(b)}{r+1}, \quad r=0,1, \ldots, 2 j-1 .
$$

Then $S_{n}^{j j a b}$ is a quadrature rule with convergence of order $2 j+1+\min \{0, \gamma\}$ for $g$, where $g(x)=$ $x^{\gamma} f(x)$, with $f \in C^{p}([0,1])$ and $p \geq 2 j$. Moreover,

$$
\begin{aligned}
S_{n}^{j j a b}(g)- & \int_{0}^{1} g(x) d x \sim \\
& \begin{cases}h^{\gamma+2 j+1} \frac{f^{(2 j)}(0)}{(2 j) !}\left\{\sum_{i=1}^{j} u_{i} v_{i}^{\gamma+2 j}+\zeta(-\gamma-2 j, a)\right\} & \gamma<0, f^{(2 j)}(0) \neq 0, \\
h^{2 j+1} \frac{g^{(2 j)}(1)}{(2 j) !}\left\{\sum_{i=1}^{j} w_{i} x_{i}^{2 j}-\frac{B_{2 j+1}(b)}{2 j+1}\right\} & \gamma>0, g^{(2 j)}(1) \neq 0,\end{cases}
\end{aligned}
$$

as $n \rightarrow \infty$.

Corollary 3.6. Suppose the nodes $u_{1}, \ldots, u_{j}$ and the weights $v_{1}, \ldots, v_{j}$ satisfy the equations

$$
\begin{aligned}
\sum_{i=1}^{j} u_{i} v_{i}{ }^{\gamma+r}=-\zeta(-\gamma-r, a), & r=0,1, \ldots, j-1, \\
\sum_{i=1}^{j} u_{i} v_{i}{ }^{r}=\frac{B_{r+1}(a)}{r+1}, & r=0,1, \ldots, j-1,
\end{aligned}
$$

and the nodes $x_{1}, \ldots, x_{k}$ and weights $w_{1}, \ldots, w_{k}$ satisfy the equations

$$
\sum_{i=1}^{k} w_{i} x_{i}^{r}=\frac{B_{r+1}(b)}{r+1}, \quad r=0,1, \ldots, 2 k-1 .
$$

Then $S_{n}^{j k a b}$ is a quadrature rule with convergence of order $\min \{j+1, \gamma+j+1,2 k+1\}$ for $g$, where $g(x)=x^{\gamma} \phi(x)+\psi(x)$, with $\phi, \psi \in C^{p}([0,1])$ and $p \geq \min \{j, 2 k\}$.

In Corollaries 3.5 and 3.6 an even number of constraints on the nodes and weights at both ends of the interval are considered. Clearly, there are analogous quadrature rules arising from an odd number of constraints at one or both ends; these are similar and explicit presentation of them is omitted. We now consider a different type of singularity. 
Theorem 3.7. Suppose $g(x)=f(x) \log x$, where $f \in C^{p}([0,1])$. The asymptotic expansion of $S_{n}^{j k a b}(g)$ as $n \rightarrow \infty$ is given by the formula

$$
\begin{aligned}
S_{n}^{j k a b}(g)= & \int_{0}^{1} g(x) d x \\
& +\sum_{r=0}^{p-1} h^{r+1} \frac{f^{(r)}(0)}{r !}\left\{\sum_{i=1}^{j} u_{i} v_{i}^{r} \log \left(v_{j} h\right)-\zeta^{\prime}(-r, a)-\frac{B_{r+1}(a)}{r+1} \log h\right\} \\
& \quad+\sum_{r=0}^{p-1} h^{r+1} \frac{(-1)^{r} g^{(r)}(1)}{r !}\left\{\sum_{i=1}^{k} w_{i} x_{i}^{r}-\frac{B_{r+1}(b)}{r+1}\right\}+O\left(h^{p+1} \log h\right),
\end{aligned}
$$

where $\zeta^{\prime}$ denotes the derivative of $\zeta$ with respect to its first argument.

Proof. This asymptotic expansion is derived from that of Theorem 3.4 by differentiating (27) with respect to $\gamma$ and evaluating the result at $\gamma=0$.

Corollary 3.8. Suppose the nodes $u_{1}, \ldots, u_{j}$ and the weights $v_{1}, \ldots, v_{j}$ satisfy the equations

$$
\begin{aligned}
\sum_{i=1}^{j} u_{i} v_{i}^{r} \log v_{i} & =\zeta^{\prime}(-r, a), & r & =0,1, \ldots, j-1, \\
\sum_{i=1}^{j} u_{i} v_{i}^{r} & =\frac{B_{r+1}(a)}{r+1}, & r & =0,1, \ldots, j-1,
\end{aligned}
$$

and the nodes $x_{1}, \ldots, x_{k}$ and weights $w_{1}, \ldots, w_{k}$ satisfy the equations

$$
\sum_{i=1}^{k} w_{i} x_{i}{ }^{r}=\frac{B_{r+1}(b)}{r+1}, \quad r=0,1, \ldots, 2 k-1 .
$$

Then $S_{n}^{j k a b}$ is a quadrature rule with error of order $O\left(\min \left\{h^{j+1} \log h, h^{2 k+1}\right\}\right)$ for $g$, where $g(x)=$ $\phi(x) \log x+\psi(x)$, with $\phi, \psi \in C^{p}([0,1])$ and $p \geq \min \{j, 2 k\}$.

3.3. Improper Integrals. For $j, n$ positive integers, we define a linear operator $R_{n}^{j}$ on $C([n, \infty))$, depending on nodes $x_{1}, \ldots, x_{j}$ and weights $w_{1}, \ldots, w_{j}$, by the formula

$$
R_{n}^{j}(g)=\sum_{k=1}^{j} w_{k} g\left(n+x_{k}\right) \text {. }
$$

Theorem 3.9. Suppose that $g(x)=e^{i \gamma x} f(x)$, where $\gamma \in \mathbb{R}, \gamma \neq 0$, and $f \in C^{p}([1, \infty)$ ), and that there exist positive constants $\beta, \alpha_{0}, \ldots, \alpha_{p}$, such that

$$
\left|f^{(r)}(x)\right|<\frac{\alpha_{r}}{x^{\beta+r}} \text { for } x>1, \quad r=0, \ldots, p .
$$

The asymptotic expansion of $R_{n}^{j}(g)$ as $n \rightarrow \infty$ is given by the formula

$$
\begin{aligned}
R_{n}^{j}(g)=\int_{n}^{\infty} g(x) d x & \\
& +e^{i \gamma n} \sum_{r=0}^{p-1} \frac{f^{(r)}(n)}{r !}\left\{\sum_{k=1}^{j} w_{k} x_{k}^{r} e^{i \gamma x_{k}}-r !\left(\frac{i}{\gamma}\right)^{r+1}\right\}+O\left(n^{-p}\right) .
\end{aligned}
$$


Proof. We integrate by parts repeatedly to obtain

$$
\int_{n}^{\infty} e^{i \gamma x} f(x) d x=e^{i \gamma n} \sum_{r=0}^{p-1}\left(\frac{i}{\gamma}\right)^{r+1} f^{(r)}(n)+\int_{n}^{\infty} e^{i \gamma x}\left(\frac{i}{\gamma}\right)^{p} f^{(p)}(x) d x,
$$

and in (41) we compute the Taylor expansion of $f$ about $x_{k}=0$ to get

$$
R_{n}^{j}(g)=e^{i \gamma n} \sum_{k=1}^{j} e^{i \gamma x_{k}} w_{k}\left\{\sum_{r=0}^{p-1} \frac{f^{(r)}(n)}{r !} x_{k}^{r}+\frac{f^{(p)}\left(n+\xi_{i} k\right)}{p !} x_{k}{ }^{p}\right\},
$$

where $\xi_{k}$ lies between 0 and $x_{k}$ for $k=1, \ldots, j$. Now combining (42), (44), and (45) we obtain (43).

Example. The function $f$ defined by the formula

$$
f(x)=\sum_{r=0}^{\infty} \frac{a_{r}}{x^{\beta+r}}
$$

with $\sum\left|a_{r}\right|<\infty$, satisfies the assumptions of Theorem 3.9 for every positive integer $p$. We remark that Theorem 3.9 can, in some instances, be generalized to $\gamma=0$, but the corresponding asymptotic expansion depends on a more detailed knowledge of $f$. For $f$ given by (46), for example, the quadrature nodes and weights for $\gamma=0$ depend on $\beta$.

Corollary 3.10. Suppose $f \in C^{p}([1, \infty)), f$ satisfies (42) for $x \in \mathbb{R}$, and $f$ is analytic in the halfplane $\operatorname{Re}(x)>$ a for some $a \in \mathbb{R}$ Suppose further that $v_{1}, \ldots, v_{j}$ are the roots of the Laguerre polynomial $L_{j}$ of degree $j$, that coefficients $u_{1}, \ldots, u_{j}$ satisfy the equations

$$
\sum_{k=1}^{j} u_{k} v_{k}^{r} e^{-v_{k}}=r !, \quad r=0,1, \ldots, j-1,
$$

and that the operator $R_{n}^{j}$ is defined with nodes $x_{k}=(i / \gamma) v_{k}$ and weights $w_{k}=(i / \gamma) u_{k}$ for $k=$ $1, \ldots, j$. Suppose finally that $\hat{T}_{m}^{j a}$ is defined to be the quadrature rule $T_{m}^{j a}$ with nodes and weights satisfying (22), but translated and scaled to the interval $[1, n]$. Then for $p \geq 2 j$, the expression $\hat{T}_{(n-1) n}^{j a}(g)+R_{n}^{j}(g)$ is an approximation for the integral $\int_{1}^{\infty} g(x) d x$, where $g(x)=e^{i \gamma x} f(x)$, with error of order $O\left(n^{-2 j}\right)$ as $n \rightarrow \infty$.

Proof. This result is just a combination of the quadrature rule of Corollary 3.2 , for the interval $[1, n]$, with the asymptotic expansion of Theorem 3.9 , for the interval $[n, \infty)$, provided

$$
\sum_{k=1}^{j} u_{k} v_{k}^{r} e^{-v_{k}}=r !, \quad r=0,1, \ldots, 2 j-1 .
$$

But (48) follows from (47), the equations

$$
\begin{gathered}
r !=\int_{0}^{\infty} x^{r} e^{-x} d x, \quad r=0,1, \ldots, \\
\int_{0}^{\infty} L_{j}(x) L_{k}(x) e^{-x} d x=0 \quad \text { for } j \neq k,
\end{gathered}
$$

(see, for example, [7] 6.1.1 and 22.2.13) and the fact that Gaussian quadratures, which are exact for polynomials of degree less than twice the number of nodes, have nodes that coincide with the roots of the corresponding orthogonal polynomials (see Section 2.4). 
We have completed the definition of the new quadratures, along with the demonstration of their asymptotic performance. We shall see that the existence of these rules, which depends on the solvability of the nonlinear systems of equations that define the nodes and weights, is assured by the theory of Chebyshev systems. The uniqueness of the rules is similarly assured. These issues of existence and uniqueness are treated next.

\section{EXISTENCE AND UNIQUENESS}

4.1. Chebyshev Systems. Material of this subsection is taken, with minor changes, from Karlin and Studden [12]. Suppose $I$ is an interval of $\mathbb{R}$, possibly infinite. A collection of $n$ real-valued continuous functions $f_{1}, \ldots, f_{n}$ defined on $I$ is a Chebyshev system if any linear combination

$$
f(x)=\sum_{i=1}^{n} a_{i} f_{i}(x),
$$

with $a_{i}$ not all zero, has at most $n-1$ zeros on $I$. This condition is equivalent to the statement that for distinct $x_{1}, \ldots, x_{n}$ in $I$,

$$
\operatorname{det}\left(\begin{array}{ccc}
f_{1}\left(x_{1}\right) & \cdots & f_{n}\left(x_{1}\right) \\
\vdots & \ddots & \vdots \\
f_{1}\left(x_{n}\right) & \cdots & f_{n}\left(x_{n}\right)
\end{array}\right) \neq 0
$$

The Chebyshev property is a characteristic of the space, rather than the basis: if $f_{1}, \ldots, f_{n}$ is a Chebyshev system, then so is any other basis of $\operatorname{span}\left\{f_{1}, \ldots, f_{n}\right\}$. If $u$ is a continuous, positive function on $I$ then scaling by $u$ preserves a Chebyshev system. Finally, if $u$ is strictly increasing and continuous on interval $J$ with range $I$, then $f_{1} \circ u, \ldots, f_{n} \circ u$ is a Chebyshev system on $J$ if and only if $f_{1}, \ldots, f_{n}$ is on $I$. (Here $f_{i} \circ u$ denotes the composition $u$ followed by $f_{i}$.)

The best-known example of a Chebyshev system is the set of polynomials

$$
1, x, \ldots, x^{n-1}
$$

on any interval $I \subset \mathbb{R}$ We shall be concerned also with the Chebyshev systems

$$
\begin{gathered}
1, x^{\gamma}, x, x^{\gamma+1}, \ldots, x^{(n-1) / 2}, x^{\gamma+(n-1) / 2} \\
1, \log x, x, x \log x, \ldots, x^{(n-1) / 2}, x^{(n-1) / 2} \log x
\end{gathered}
$$

on $I=(0, a]$, where $\gamma \in \mathbb{R} \backslash \mathbb{Z}$ and $a>0$. These systems are special cases of the system of Müntz functions (see, for example, Borwein and Erdélyi [13] p. 133)

$$
M=\left\{x^{\gamma_{i}} \log ^{k} x \mid k=0, \ldots, n_{i}-1, i=1, \ldots, j\right\}
$$

on $I=(0, \infty)$, where $\gamma_{1}, \ldots, \gamma_{j}$ are distinct real numbers and $n_{1}, \ldots, n_{j}$ are positive integers with $\sum n_{i}=n$. To see this is a Chebyshev system, suppose $f \in$ span $M$ and use induction in $n$ on $(d / d \log x)\left[f(x) x^{-\gamma_{j}}\right]$, in combination with Rolle's theorem. Another Chebyshev system that will arise is the system

$$
L=\left\{\left(x+\gamma_{i}\right)^{-k} \mid k=1, \ldots, n_{i}, i=1, \ldots, j\right\}
$$

on $I=[0, \infty)$, where $\gamma_{1}, \ldots, \gamma_{j}$ are distinct positive real numbers and $n_{1}, \ldots, n_{j}$ are positive integers with $\sum n_{i}=n$. This is indeed a Chebyshev system, for if $f \in \operatorname{span} L$ then the function $f(x) \prod_{i=1}^{j}\left(x+\gamma_{i}\right)^{n_{i}}$ is a polynomial in $x$ of degree $n-1$. 
Suppose $f_{1}, \ldots, f_{n}$ is a Chebyshev system on the interval $I$. The moment space $\mathcal{M}_{n}$ with respect to $f_{1}, \ldots, f_{n}$ is the set

$$
\mathcal{M}_{n}=\left\{\mathbf{c}=\left(c_{1}, \ldots, c_{n}\right) \in \mathbb{R}^{n} \mid c_{i}=\int_{l} f_{i}(x) d \sigma(x), i=1, \ldots, n\right\},
$$

where the measure $\sigma$ ranges over the set of nondecreasing right-continuous functions of bounded variation on $I$. It can be shown that $\mathcal{M}_{n}$ is the convex cone associated with points in the curve $C$, where

$$
C=\left\{\left(f_{1}(x), \ldots, f_{n}(x)\right) \mid x \in I\right\} .
$$

In other words, $\mathcal{M}_{n}$ can be represented as

$$
\mathcal{M}_{n}=\left\{c=\sum_{j=1}^{p} \alpha_{j} y_{j} \mid \alpha_{j}>0, y_{j} \in C, j=1, \ldots, p, p \geq 1\right\} .
$$

The index $\mathcal{I}(\mathrm{c})$ of a point $\mathrm{c}$ of $\mathcal{M}_{n}$ is the minimum number of points of $C$ that can be used in the representation of $\mathbf{c}$, under the convention that a point $\left(f_{1}(x), \ldots, f_{n}(x)\right)$ is counted as a half point if $x$ is from the boundary of $I$ and receives a full count otherwise. The index of a quadrature involving $x_{1}, \ldots, x_{p}$ is determined by counting likewise.

Proofs of the next three theorems are somewhat elaborate and are omitted here; they can be found in Karlin and Studden [12].

Theorem 4.1 ([12] p. 42). Suppose $I=[a, b]$ is a closed interval. A point $\mathbf{c} \in \mathcal{M}_{n}, \mathbf{c} \neq \mathbf{0}$, is a boundary point of $\mathcal{M}_{n}$ if and only if $\mathcal{I}(\mathbf{c})<n / 2$. Moreover, if $\sigma$ is a measure corresponding to a boundary point $\mathrm{c} \in \mathcal{M}_{n}$, then there is a unique quadrature

$$
\sum_{i=1}^{p} w_{i} f_{r}\left(x_{i}\right)=\int_{l} f_{r}(x) d \sigma(x), \quad r=1, \ldots, n,
$$

where $p \leq(n+1) / 2, a \leq x_{1}<x_{2}<\cdots<x_{p} \leq b$, and $w_{i}>0, i=1, \ldots, p$.

Theorem 4.2 ([12] p. 47). Suppose $I=[a, b]$ is a closed interval. Any point $\mathbf{c}$ in the interior of $\mathcal{M}_{n}$ satisfies $\mathcal{I}(\mathbf{c})=n / 2$. Moreover, if $\sigma$ is a measure corresponding to $\mathbf{c}$, then there are exactly two quadratures

$$
\sum_{i=1}^{p} w_{i} f_{r}\left(x_{i}\right)=\int_{l} f_{r}(x) d \sigma(x), \quad r=1, \ldots, n,
$$

of index $n / 2$, where $w_{i}>0, i=1, \ldots, p$. In particular, if $n=2 m$, then $p=m$ or $p=m+1$ and

$$
\begin{aligned}
& a<x_{1}<x_{2}<\cdots<x_{m}<b \text { or } \\
& a=x_{1}<x_{2}<\cdots<x_{m+1}=b ;
\end{aligned}
$$

if $n=2 m+1$, then $p=m+1$ and

$$
\begin{aligned}
& a=x_{1}<x_{2}<\cdots<x_{m+1}<b \text { or } \\
& a<x_{1}<x_{2}<\cdots<x_{m+1}=b .
\end{aligned}
$$

Theorem $4.3\left([12]\right.$ p. 65). Let $\mathcal{P}_{n}$ denote the nonnegative linear combinations of $f_{1}, \ldots, f_{n}$,

$$
\mathcal{P}_{n}=\left\{f \mid f(x)=\sum_{i=1}^{n} a_{i} f_{i}(x) \text { and } f(x) \geq 0 \text { for all } x \in I\right\} .
$$


The point $\mathbf{c}=\left(c_{1}, \ldots, c_{n}\right)$ is an element of $\mathcal{M}_{n}$ if and only if

$$
\sum_{i=1}^{n} a_{i} f_{i} \in \mathcal{P}_{n} \quad \text { implies } \quad \sum_{i=1}^{n} a_{i} c_{i} \geq 0 .
$$

Moreover, $\mathbf{c}$ is in the interior of $\mathcal{M}_{n}$ if and only if

$$
\sum_{i=1}^{n} a_{i} f_{i} \in \mathcal{P}_{n} \text { and } \sum_{i=1}^{n} a_{i}{ }^{2}>0 \text { imply } \sum_{i=1}^{n} a_{i} c_{i}>0 .
$$

Theorem 4.4 ([12] p. 106). Suppose $f_{i}(x)=x^{i-1}$ for $i=1, \ldots, n$, and $I=[a, b]$. If $n=2 m$, then $\mathbf{c}=\left(c_{1}, \ldots, c_{n}\right)$ is an element of $\mathcal{M}_{n}$ if and only if the two quadratic forms

$$
\sum_{i, j=1}^{m}\left[c_{i+j}-a c_{i+j-1}\right] \alpha_{i} \alpha_{j} \text { and } \sum_{i, j=1}^{m}\left[b c_{i+j-1}-c_{i+j}\right] \beta_{i} \beta_{j}
$$

are nonnegative definite. If $n=2 m+1$, then $\mathrm{c} \in \mathcal{M}_{n}$ if and only if the two quadratic forms

$$
\sum_{i, j=1}^{m+1} c_{i+j-1} \alpha_{i} \alpha_{j} \text { and } \sum_{i, j=1}^{m}\left[(a+b) c_{i+j}-a b c_{i+j-1}-c_{i+j+1}\right] \beta_{i} \beta_{j}
$$

are nonnegative definite. Moreover, for either parity of $n, \mathrm{c}$ is in the interior of $\mathcal{M}_{n}$ if and only if the corresponding quadratic forms are both positive definite.

Proof. A theorem of Lukács (see, for example, Szegö [11] p. 4) states that a polynomial $f$ of degree $n-1$ that is nonnegative on $[a, b]$ can be represented in the form

$$
f(x)= \begin{cases}(x-a) p(x)^{2}+(b-x) q(x)^{2} & n=2 m \\ p(x)^{2}+(b-x)(x-a) q(x)^{2} & n=2 m+1\end{cases}
$$

where $p$ and $q$ are polynomials such that the degree of each term in (64) does not exceed $n-1$. The combination of (64) and Theorem 4.3 proves the theorem.

4.2. Müntz System Quadratures. The systems of equations (22), (24), (36), and (39) that define the quadrature rules of Section 3 are special cases of the system of equations

$$
\sum_{m=1}^{i n / 2\rceil} w_{m} x_{m}^{\gamma_{i}} \log ^{k} x_{m}=(-1)^{k+1} \zeta^{(k)}\left(-\gamma_{i}, a\right), \quad k=0, \ldots, n_{i}-1, i=1, \ldots, j,
$$

for distinct real numbers $\gamma_{1}, \ldots, \gamma_{j}$ and positive integers $n_{1}, \ldots, n_{j}$ with $\sum n_{i}=n$. Here $\zeta^{(k)}$ denotes the $k$ th derivative of $\zeta$ with respect to its first argument. The existence and uniqueness of the solution of (65) follow from the existence and uniqueness of quadratures for Chebyshev systems, once it is established that there is a measure $\sigma_{a}$ with

$$
\int_{0}^{a} x^{\gamma_{i}} \log ^{k} x d \sigma_{a}(x)=(-1)^{k+1} \zeta^{(k)}\left(-\gamma_{i}, a\right), \quad k=0, \ldots, n_{i}-1, i=1, \ldots, j,
$$

in other words, that the moment space $\mathcal{M}_{n}$ of the Chebyshev system of Müntz functions

$$
M=\left\{x^{\gamma_{i}} \log ^{k} x \mid k=0, \ldots, n_{i}-1, i=1, \ldots, j\right\},
$$

on $(0, a]$ contains the point

$$
\mathbf{c}=\left(-\zeta\left(-\gamma_{1}, a\right), \ldots,(-1)^{n_{j}} \zeta^{\left(n_{j}-1\right)}\left(-\gamma_{j}, a\right)\right) .
$$


We will show that this condition is satisfied provided that $a$ is sufficiently large. It would be convenient to have tight bounds for $a$, in particular for systems (22), (24), (36), and (39), but it appears that such bounds are difficult to obtain. Even for the regular cases (22) and (24), where by Theorem 4.4 the existence of $\sigma_{a}$ is equivalent to the positive definiteness of two matrices, precise bounds for arbitrary $j$ appear difficult. (Numerical examples below provide evidence that $a / j$ may be chosen as small as 5/6.)

Theorem 4.5. Suppose $\gamma_{1}, \ldots, \gamma_{j}$ are distinct real numbers, each greater than -1 , and $n_{1}, \ldots, n_{j}$ are positive integers with $\sum n_{i}=n$. Then for sufficiently large a there exists a measure $\sigma_{a}$ such that the system of equations (66) is satisfied and $\mathbf{c}$ defined by (68) is in the interior of the moment space $\mathcal{M}_{n}$.

Proof. We construct a continuous weight function $\sigma_{a}^{\prime}$ satisfying (66) and show that for sufficiently large $a, \sigma_{a}^{\prime}(x)$ is positive for $x \in[0, a]$.

We linearly combine the equations of (66) to obtain the equivalent system

$$
\begin{aligned}
\int_{0}^{a}(x / a)^{\gamma_{i}} \log ^{k}(x / a) d \sigma_{a}(x)=(-1)^{k+1} \sum_{r=0}^{k}\left(\begin{array}{l}
k \\
r
\end{array}\right) \frac{\zeta^{(r)}\left(-\gamma_{i}, a\right)}{a^{\gamma_{i}}} \log ^{k-r} a, \\
\quad k=0, \ldots, n_{i}-1, i=1, \ldots, j,
\end{aligned}
$$

where we have used the binomial theorem to expand $\log ^{k}(x / a)=(\log x-\log a)^{k}$. We define the weight $\sigma_{a}^{\prime}$ by the formula

$$
\sigma_{a}^{\prime}(x)=\sum_{m=0}^{n-1} \alpha_{m, a}(x / a)^{m}
$$

and combine (69), (70), and the equalities

$$
\int_{0}^{1} x^{\gamma} \log ^{k} x d x=\frac{(-1)^{k} k !}{(1+\gamma)^{k+1}}, \quad \gamma>-1, k=0,1, \ldots
$$

to obtain the equations in $\alpha_{0, a}, \ldots, \alpha_{n-1, a}$

$$
\begin{aligned}
\sum_{m=0}^{n-1} \frac{\alpha_{m, a}(-1)^{k} k !}{\left(1+\gamma_{i}+m\right)^{k+1}}=(-1)^{k+1} \sum_{r=0}^{k}\left(\begin{array}{l}
k \\
r
\end{array}\right) \frac{\zeta^{(r)}\left(-\gamma_{i}, a\right)}{a^{1+\gamma_{i}}} \log ^{k-r} a, \\
\quad k=0, \ldots, n_{i}-1, i=1, \ldots, j .
\end{aligned}
$$

This $n$-dimensional linear system is nonsingular, since the set of functions

$$
\left\{\left(x+\gamma_{i}+1\right)^{-k} \mid k=1, \ldots, n_{i}, i=1, \ldots, j\right\}
$$

forms a Chebyshev system on $[0, \infty)$, as established at (51). Thus (71) possesses a unique solution $\alpha_{0, a}, \ldots, \alpha_{n-1, a}$.

We now determine

$$
\alpha_{m}=\lim _{a \rightarrow \infty} \alpha_{m, a}, \quad m=0, \ldots n-1
$$


The asymptotic expansion of $\zeta^{(r)}\left(-\gamma_{i}, a\right)$ as $a \rightarrow \infty$ can be derived by differentiating (10); the first several terms are given by

$$
\zeta^{(r)}\left(-\gamma_{i}, a\right)=a^{1+\gamma_{i}} \sum_{l=0}^{r}\left(\begin{array}{l}
r \\
l
\end{array}\right) \frac{(-1)^{l+1}(r-l) ! \log ^{l} a}{\left(1+\gamma_{i}\right)^{r-l+1}}+O\left(a^{\gamma_{i}} \log ^{r} a\right) .
$$

Combining (71) and (73), changing the order of summation, and twice applying the product differentiation rule

$$
\frac{d^{r}}{d \gamma^{r}}(f(\gamma) g(\gamma))=\sum_{s=0}^{r}\left(\begin{array}{l}
r \\
s
\end{array}\right) f^{(s)}(\gamma) g^{(r-s)}(\gamma)
$$

we obtain

$$
\sum_{m=0}^{n} \frac{\alpha_{m}(-1)^{k} k !}{\left(1+\gamma_{i}+m\right)^{k+1}}=\frac{(-1)^{k} k !}{\left(1+\gamma_{i}\right)^{k+1}}, \quad k=0, \ldots, n_{i}-1, i=1, \ldots, j,
$$

which immediately reduces to

$$
\alpha_{m}= \begin{cases}1 & m=0 \\ 0 & m=1, \ldots, n-1 .\end{cases}
$$

The combination of (70), (72), and (74) gives

$$
\lim _{a \rightarrow \infty} \sigma_{a}^{\prime}(a x)=1, \quad x \in[0,1],
$$

which implies that for $a$ sufficiently large, $\sigma_{a}^{\prime}(x)>0$ for $x \in[0, a]$. The point $c$ defined by (68) is in the interior of $\mathcal{M}_{n}$, since small perturbations of $\mathrm{c}$ will preserve the positivity of $\sigma_{a}^{\prime}$.

Theorem 4.2 of Section 4.1 ensures the existence of Gaussian quadratures for a Chebyshev system $f_{1}, \ldots, f_{n}$ defined on an interval $I$, under the assumption that $I$ is closed, whereas the system $M$ of (67) is Chebyshev on $I=(0, a]$. As a consequence, we require the following result.

Theorem 4.6. Suppose the collection of functions $f_{1}, \ldots, f_{n}$ forms Chebyshev system on $I=(a, b]$ and each is integrable on $[a, b]$ with respect to a measure $\sigma$ corresponding to a point $\mathbf{c}$ in the interior of $\mathcal{M}_{n}$. Then there exists a unique quadrature

$$
\sum_{i=1}^{p} w_{i} f_{r}\left(x_{i}\right)=\int_{a}^{b} f_{r}(x) d \sigma(x), \quad r=1, \ldots, n,
$$

of index $n / 2$, where $w_{i}>0$ and $x_{i} \in I, i=1, \ldots, p$. In particular, if $n=2 m$, then $p=m$ and

$$
a<x_{1}<x_{2}<\cdots<x_{m}<b
$$

if $n=2 m+1$, then $p=m+1$ and

$$
a<x_{1}<x_{2}<\cdots<x_{m+1}=b .
$$

Proof. The Chebyshev property implies that there exists $\delta$ with $a<\delta<b$ such that $f_{i}$ is nonzero on $(a, \delta], i=1, \ldots, n$. We define the function $u$ on $I$ by the formula

$$
u(x)= \begin{cases}\max _{i=1, \ldots, n}\left|f_{i}(x)\right| & x \in(a, \delta], \\ u(\delta) & x \in(\delta, b]\end{cases}
$$


and observe that $u$ is continuous and positive on $I$ and integrable on $[a, b]$ with respect to $\sigma$. Now we define functions $g_{1}, \ldots, g_{n}$ on $[a, b]$ by the formula

$$
g_{i}(x)=\left\{\begin{array}{ll}
\frac{f_{i}(x)}{u(x)} & x \in(a, b], \\
\lim _{x \rightarrow a} g_{i}(x) & x=a,
\end{array} \quad i=1, \ldots, n .\right.
$$

The system $g_{1}, \ldots, g_{n}$ is a Chebyshev system on $[a, b]$ and is integrable with respect to the measure $\int_{a}^{x} u(t) d \sigma(t)$. Theorem 4.2 therefore is applicable and assures the existence of exactly two quadratures of index $n / 2$ for the interval $[a, b]$, one of which includes the point $x_{1}=a$. Our assumption $x_{i} \in(a, b]$ excludes this case and we are left with the single quadrature presented in (76) and (77).

The next theorem, which is the principal analytical result of this section, follows directly from Theorems 4.5 and 4.6. The existence and uniqueness of the quadratures defined in Section 3 follow from it. It also hints at the existence of somewhat more general quadratures, for singularities of the form $x^{\gamma} \log ^{k} x$, but we do not evaluate these here.

Theorem 4.7. Suppose $\gamma_{1}, \ldots, \gamma_{j}$ are distinct real numbers, each greater than -1 , and $n_{1}, \ldots, n_{j}$ are positive integers with $\sum n_{i}=n$. For sufficiently large $a$, the system of equations

$$
\sum_{m=1}^{[n / 2\rceil} w_{m} x_{m}^{\gamma_{i}} \log ^{k} x_{m}=(-1)^{k+1} \zeta^{(k)}\left(-\gamma_{i}, a\right), \quad k=0, \ldots, n_{i}-1, i=1, \ldots, j,
$$

has a unique solution $w_{1}, \ldots, w_{n}, x_{1}, \ldots, x_{n}$ satisfying $w_{i}>0, i=1, \ldots,\lceil n / 2\rceil$, and $0<x_{1}<$ $\ldots<x_{\lceil n / 2\rceil} \leq a$, with $x_{\lceil n / 2\rceil}=a$ if $n$ is odd.

\section{COMPUTATION OF THE NODES AND WEIGHTS}

The nodes and weights of the quadratures defined in Section 3 are computed by numerically solving the nonlinear systems (22), (24), (36), and (39). Conventional techniques for this problem are either overly cumbersome or converge too slowly to be practical. Recently, Ma, Rokhlin, and Wandzura [6] addressed this need by developing a practical numerical algorithm that is effective in a fairly general setting. They construct a simplified Newton's method and combine it with a continuation (homotopy) method. We present their method in an abbreviated form in Section 5.2; the reader is referred to [6] for more detail.

The systems for regular integrands, however, can be solved even more simply, as we see next.

5.1. Regular Integrands. The classical theory of Gaussian quadratures for polynomials, summarized in Section 2.4, can be exploited to solve (22) and (24). In particular, suppose that $p_{0}, \ldots, p_{j}$ are the orthogonal polynomials on $[0, a]$, given by the recurrence (13) and (14), under the assumption that

$$
\int_{0}^{a} x^{r} \omega(x) d x=\frac{B_{r+1}(a)}{r+1}, \quad r=0, \ldots, 2 j-1 .
$$

Then the roots $x_{1}, \ldots, x_{j}$ of $p_{j}$ and corresponding Christoffel numbers $w_{1}, \ldots, w_{j}$ satisfy (22). The polynomials $p_{1}, \ldots, p_{j}$ can be calculated in symbolic form; their coefficients are rational if $a$ is rational. The roots of $p_{j}$ can be computed by Newton iteration and the Christoffel numbers can be obtained 
using (16). Similar treatment can be applied to the system (24) containing an odd number of equations, using the interval $[0, a-1]$, under the assumption

$$
\int_{0}^{a-1} x^{r} \tau(x) d x=\frac{B_{r+1}(a)}{r+1}, \quad r=0, \ldots, 2 j-2 .
$$

The Gauss-Radau quadrature is computed using the formula (18) for the modified Christoffel numbers. For these tasks it is convenient to use a software system that can manipulate polynomials with fullprecision rational coefficients. The author implemented code for these computations in Pari/GP [14].

It should be noted that the proposed procedure is suitable for relatively small values of $j$ (less than, say, 20). It is neither very efficient nor very stable, but was quite adequate for our purposes. (Unlike the situation for standard Gaussian quadratures, where the number of nodes depends on the size of the problem, here $j$ depends only on the desired order of convergence.) If it is required to compute the nodes and weights of (22) or (24) for large $j$, the reader may consider numerical schemes for Gaussian quadrature proposed by other authors, for example, that of Gautschi [15] or Golub and Welsch [16].

5.2. Singular Integrands. The systems (36) and (39) for singular integrands cannot be solved using methods for standard Gaussian quadrature, since the nodes to be computed do not coincide with the roots of any closely-related orthogonal polynomials. We employ instead the algorithm for such systems developed by Ma, Rokhlin, and Wandzura [6], which we now describe.

A collection of $2 n$ real-valued continuously differentiable functions $f_{1}, \ldots, f_{2 n}$ defined on an interval $I=[a, b]$ is an Hermite system if

$$
\operatorname{det}\left(\begin{array}{cccc}
f_{1}\left(x_{1}\right) & f_{2}\left(x_{1}\right) & \cdots & f_{2 n}\left(x_{1}\right) \\
f_{1}^{\prime}\left(x_{1}\right) & f_{2}^{\prime}\left(x_{1}\right) & \cdots & f_{2 n}^{\prime}\left(x_{1}\right) \\
f_{1}\left(x_{2}\right) & f_{2}\left(x_{2}\right) & \cdots & f_{2 n}\left(x_{2}\right) \\
f_{1}^{\prime}\left(x_{2}\right) & f_{2}^{\prime}\left(x_{2}\right) & \cdots & f_{2 n}^{\prime}\left(x_{2}\right) \\
\vdots & \vdots & & \vdots \\
f_{1}\left(x_{n}\right) & f_{2}\left(x_{n}\right) & \cdots & f_{2 n}\left(x_{n}\right) \\
f_{1}^{\prime}\left(x_{n}\right) & f_{2}^{\prime}\left(x_{n}\right) & \cdots & f_{2 n}^{\prime}\left(x_{n}\right)
\end{array}\right) \neq 0
$$

for any choice of distinct $x_{1}, \ldots, x_{n}$ on $I$. An Hermite system that is also a Chebyshev system is an extended Hermite system. The following theorem is a direct consequence of the definition; the proofs of the subsequent two theorems are contained in [6].

Theorem 5.1. Suppose that the functions $f_{1}, \ldots, f_{2 n}$ constitute an Hermite system on the interval $[a, b]$ and $x_{1}, \ldots, x_{n}$ are $n$ distinct points on $[a, b]$. Then there exist unique coefficients $\alpha_{i j}, \beta_{i j}, i=$ $1, \ldots, n, j=1, \ldots, 2 n$, such that

$$
\begin{array}{ll}
\sigma_{i}\left(x_{k}\right)=0 & \eta_{i}\left(x_{k}\right)=\delta_{i k} \\
\sigma_{i}^{\prime}\left(x_{k}\right)=\delta_{i k} & \eta_{i}^{\prime}\left(x_{k}\right)=0
\end{array}
$$

for $i=1, k=1, \ldots, n$, where the functions $\sigma_{i}, \eta_{i}$ are defined by the formulae

$$
\begin{aligned}
& \sigma_{i}(x)=\sum_{j=1}^{2 n} \alpha_{i j} f_{j}(x) \\
& \eta_{i}(x)=\sum_{j=1}^{2 n} \beta_{i j} f_{j}(x)
\end{aligned}
$$

for $i=1, \ldots, n$. 
Theorem 5.2 ([6] p. 979). Suppose that the functions $f_{1}, \ldots, f_{2 n}$ are an Hermite system on $[a, b]$. Suppose further that $S \subset[a, b]^{n}$ is the set of points with distinct coordinates $x_{1}, \ldots, x_{n}$. Suppose finally that the mapping $F: S \rightarrow \mathbb{R}^{n}$ is defined by the formula

$$
F\left(x_{1}, \ldots, x_{n}\right)=\left(\int_{a}^{b} \sigma_{1}(x) \omega(x) d x, \ldots, \int_{a}^{b} \sigma_{n}(x) \omega(x) d x\right),
$$

with the functions $\sigma_{1}, \ldots, \sigma_{n}$ defined by (80) through (82). Then $x_{1}, \ldots, x_{n}$ are the Gaussian nodes for the system of functions $f_{1}, \ldots, f_{2 n}$ with respect to the weight $\omega$ if and only if $F\left(x_{1}, \ldots, x_{n}\right)=\mathbf{0}$.

Theorem 5.3 ([6] p. 983). Suppose that the functions $f_{1}, \ldots, f_{2 n}$ are an extended Hermite system on $[a, b], S \subset[a, b]^{n}$ is the set of points with distinct coordinates, and the mapping $G: S \rightarrow \mathbb{R}^{n}$ is defined by the formula

$$
G\left(x_{1}, \ldots, x_{n}\right)=\left(x_{1}+\frac{\int_{a}^{b} \sigma_{1}(x) \omega(x) d x}{\int_{a}^{b} \eta_{1}(x) \omega(x) d x}, \ldots, x_{n}+\frac{\int_{a}^{b} \sigma_{n}(x) \omega(x) d x}{\int_{a}^{b} \eta_{n}(x) \omega(x) d x}\right),
$$

with the functions $\sigma_{1}, \ldots, \sigma_{n}$ and $\eta_{1}, \ldots, \eta_{n}$ defined by (80) through (83). Suppose further that $f_{i} \in$ $C^{3}((a, b))$ for $i=1, \ldots, 2 n$ and the function $F$ is defined by (84). Suppose finally that $\mathbf{x}^{*}$ is the unique zero of $F$, that $\mathbf{x}_{0}$ is an arbitrary point of $S$, and that the sequence $\mathbf{x}_{1}, \mathbf{x}_{2}, \ldots$ is defined by the formula

$$
\mathbf{x}_{i+1}=G\left(x_{i}\right), \quad i=0,1, \ldots .
$$

Then there exists $\epsilon>0$ and $\alpha>0$ such that the sequence $\mathbf{x}_{1}, \mathbf{x}_{2}, \ldots$ generated by (86) converges to $\mathbf{x}^{*}$ and

$$
\left\|\mathbf{x}_{i+1}-\mathbf{x}^{*}\right\| \leq \alpha\left\|\mathbf{x}_{i}-\mathbf{x}^{*}\right\|^{2}
$$

for any initial point $\mathbf{x}_{0}$ such that $\left\|\mathbf{x}_{0}-\mathbf{x}^{*}\right\|<\epsilon$.

The key feature of this theorem is the quadratic convergence indicated by (87). The solution $\mathbf{x}^{*}$ is obtained by an iterative procedure; each step consists of computing the coefficients that determine $\sigma_{1}, \ldots, \sigma_{n}$ and $\eta_{1}, \ldots, \eta_{n}$ by inverting the matrix of (79), then computing the integrals that define $G$ by taking linear combinations, using these coefficients, of integrals of $f_{1}, \ldots, f_{2 n}$. Theorem 5.3 ensures that with appropriate choice of starting value $\mathbf{x}_{0}$, convergence is rapid and certain.

For quadrature nodes $\mathbf{x}^{*}=\left(x_{1}, \ldots, x_{n}\right)$, the quadrature weights are given by the integrals of $\eta_{i}$, namely

$$
\left(w_{1}, \ldots, w_{n}\right)=\left(\int_{a}^{b} \eta_{1}(x) \omega(x) d x, \ldots, \int_{a}^{b} \eta_{1}(x) \omega(x) d x\right) .
$$

We note that Theorems 5.1 through 5.3 concern Gaussian quadratures with $n$ nodes and weights to integrate $2 n$ functions on the interval $[a, b]$ exactly. For Gauss-Radau quadratures, in which node $x_{n}=$ $b$ is fixed and $2 n-1$ functions are integrated exactly, only a slight change is required. In particular, functions $\sigma_{1}, \ldots, \sigma_{n-1}$ (without $\sigma_{n}$ ) and $\eta_{1}, \ldots, \eta_{n}$ are defined as before by (80) through (83), except that the summations in (82) and (83) exclude $f_{2 n}$. Their coefficients $\alpha_{i j}, i=1, \ldots, n-1, j=$ $1, \ldots, 2 n-1$, and $\beta_{i j}, i=1, \ldots, n, j=1, \ldots, 2 n-1$, are obtained by inverting the matrix which results from removing the last row and column from the matrix of (79). The revised mapping $G: S \rightarrow \mathbb{R}^{n-1}$ has $n-1$ components defined as the first $n-1$ components in (85). Finally, as before, the quadrature weights are given by (88). 
In order to obtain a sufficiently good starting estimate for the solution of $F(\mathbf{x})=0$, a continuation procedure can be used, as outlined in the following theorem.

Theorem 5.4 (see, for example, [6] p. 975). Suppose that $\mathbf{F}:[0,1] \times[a, b]^{n} \rightarrow \mathbb{R}^{n}$ is a function with a unique solution $\mathbf{x}_{t}$ to the equation $\mathbf{F}(t, \mathbf{x})=\mathbf{0}$ for all $t \in[0,1]$, suppose that $\mathbf{x}_{t}$ is a continuous function of $t$, and suppose that $\mathbf{F}(1, \mathbf{x})=F(\mathbf{x})$. Finally, suppose $\mathbf{x}_{0}$ is given and for some $\delta>0$ there is a procedure $\mathbf{P}$ to compute $\mathbf{x}_{t}$ for $t \in[0,1]$, given an estimate $\tilde{\mathbf{x}}_{t}$ with $\left|\tilde{\mathbf{x}}_{t}-\mathbf{x}_{t}\right|<\delta$. Then there exists a positive integer $m$ such that the following procedure can be used to compute the solution of $F(\mathbf{x})=\mathbf{0}$ :

$$
\text { For } i=1, \ldots, m \text {, use } \mathbf{P} \text { to compute } \mathbf{x}_{i / m} \text {, given the estimate } \mathbf{x}_{(i-1) / m} \text {. }
$$

The required solution of $F(\mathbf{x})=\mathbf{0}$ is $\mathbf{x}_{1}$.

More typically, of course, $\delta$ and any bound on $\left|\mathbf{x}_{t+\epsilon}-\mathbf{x}_{t}\right| / \epsilon$ depend on $t$ and in a practical implementation the step size is chosen adaptively.

To compute the solutions of (36) and (39), it is effective to use a continuation procedure with respect to both $j$ and $a$. Solutions for the first few values of $j$ are readily obtained without requiring good initial estimates. Given a solution of (36) for the interval $[0, a]$ with nodes $u_{1}, \ldots, u_{j}$ and weights $v_{1}, \ldots, v_{j}$, we choose an initial estimate $\tilde{u}_{1}, \ldots, \tilde{u}_{j+1}, \tilde{v}_{1}, \ldots, \tilde{v}_{j+1}$ for $j+1$ and the interval $[0, a+$ 1] defined by the formulae

$$
\tilde{u}_{i}=\left\{\begin{array}{ll}
u_{i} & i=1, \ldots, j, \\
a & i=j+1,
\end{array} \quad \tilde{v}_{i}= \begin{cases}v_{i} & i=1, \ldots, j, \\
1 & i=j+1 .\end{cases}\right.
$$

This choice exactly satisfies the equations

$$
\begin{aligned}
\sum_{i=1}^{j+1} \tilde{u}_{i} \tilde{v}_{i}^{\gamma+r}=-\zeta(-\gamma-r, a+1), & r=0,1, \ldots, j-1, \\
\sum_{i=1}^{j+1} \tilde{u}_{i} \tilde{v}_{i}^{r}=\frac{B_{r+1}(a+1)}{r+1}, & r=0,1, \ldots, j-1,
\end{aligned}
$$

as follows immediately from the difference formula (4) for $B_{n}$ and (11) for $\zeta$, but the corresponding equations for $r=j$ are not satisfied. Those equations are approximately satisfied, however, and we can start with the actual values of the sums for $r=j$ as the required values. These are then varied continuously, obtaining the corresponding solutions, until they coincide with the intended values $-\zeta(-\gamma-j, a+1)$ and $B_{j+1}(a+1) /(j+1)$. This procedure can be used without alteration for (39).

Once the solution for $j+1$ and the interval $[0, a+1]$ is obtained, $a$ can be continuously varied, in a continuation procedure, to obtain solutions for different intervals.

\section{NUMERICAL EXAMPLES}

The procedures described in Section 5 were implemented in Pari/GP [14] for both the regular cases and the singular cases. The matrix in (79), which must be inverted, is very poorly conditioned for many choices of $n, x_{1}, \ldots, x_{n}$, and $f_{1}, \ldots, f_{2 n}$. This difficulty was met by using the extended precision capability of Pari/GP. 
TABLE 1. The minimum value of $a$, considered as a function of $j$, such that the point $\left(B_{1}(a) / 1, \ldots, B_{j}(a) / j\right)$ is in the moment space $\mathcal{M}_{2 j}$ of the polynomials $1, x, \ldots, x^{2 j-1}$ on the interval $[0, a]$. The moment space is defined at (52).

\begin{tabular}{|cc|cc|rc|cc|}
\hline$j$ & $\min a$ & $j$ & $\min a$ & \multicolumn{1}{c}{$j$} & $\min a$ & $j$ & $\min a$ \\
\hline 1 & 0.78868 & 5 & 3.96696 & 9 & 7.21081 & 13 & 10.47885 \\
2 & 1.57085 & 6 & 4.77448 & 10 & 8.02618 & 14 & 11.29815 \\
3 & 2.36347 & 7 & 5.58463 & 11 & 8.84274 & 15 & 12.11815 \\
4 & 3.16288 & 8 & 6.39687 & 12 & 9.66035 & 16 & 12.93878 \\
\hline
\end{tabular}

6.1. Nodes and Weights. The nodes and weights of (22), (24), (36), and (39) that determine the quadratures of Section 3 were computed for a range of values of the parameter $j$. For each choice of $j, a$ was chosen, by experiment, to be the smallest integer leading to positive nodes and weights (see Theorem 4.7). For the regular case (22), the characterization in Theorem 4.4 was used to determine the minimum value of $a \in \mathbb{R}$, for $j=1, \ldots, 16$, that satisfies $\mathbf{c}=\left(B_{1}(a) / 1, \ldots, B_{j}(a) / j\right) \in \mathcal{M}_{2 j}$. In particular, we obtained the minimum value of $a$ such that the quadratic forms in (62) are nonnegative definite. This determination was made by calculating the determinant of each corresponding matrix symbolically and solving for the largest root of the resulting polynomial in $a$. These values are given in Table 1. This evidence suggests that $\lim _{j \rightarrow \infty} j^{-1} \min a$ exists and is roughly $5 / 6$, meaning that the number of trapezoidal nodes displaced is less than the number of Gaussian nodes replacing them, for quadrature rules of all orders. This relationship also appears to hold, to an even greater extent, for the singular cases.

The values of selected nodes and weights, for the regular cases and for singularities $x^{-1 / 2}$ and $\log x$, are presented in an appendix. Of particular simplicity are the first two rules for regular integrands,

$$
T_{n}^{1,1}(f)=h\left[\frac{1}{2} f(h / 6)+f(h)+\cdots+f(1-h)+\frac{1}{2} f(1-h / 6)\right],
$$

where $h=1 /(n+1)$, for $n=0,1, \ldots$, and

$$
\begin{aligned}
& T_{n}^{2,2}(f)=h\left[\frac{25}{48} f(h / 5)+\frac{47}{48} f(h)+f(2 h)+\cdots\right. \\
& \left.\quad+f(1-2 h)+\frac{47}{48} f(1-h)+\frac{25}{48} f(1-h / 5)\right],
\end{aligned}
$$

where $h=1 /(n+3)$, for $n=0,1, \ldots$ These rules are of third and fourth order convergence, respectively. The first is noteworthy for having the same weights as, but higher order than, the trapezoidal rule; the second has asymptotic error $1 / 4$ that of Simpson's rule with the same number of nodes.

The lowest-order rule presented for logarithmic singularities,

$$
S_{n}^{1,1,1,1}(g)=h\left[\frac{1}{2} g(h /(2 \pi))+g(h)+\cdots+g(1-h)+\frac{1}{2} g(1)\right],
$$

approximates $\int_{0}^{1} g(x) d x$ with error of order $O\left(h^{2} \log h\right)$ for $g(x)=\phi(x) \log x+\psi(x)$, provided $\phi$ and $\psi$ are regular functions on $[0,1]$. The corresponding rule for the singularity $x^{-1 / 2}$ is not quite as simple, for there the quantity $2 \pi$ in (93) is replaced with $4 \zeta(1 / 2)^{2}$. 
6.2. Quadrature Performance. To demonstrate the performance of the quadrature rules, they were used in a Fortran routine (with real $* 8$ arithmetic) to numerically compute the integrals

$$
\int_{0}^{1}[\cos (200 x) s(x)+\cos (200 x+.3)] d x
$$

for the functions $s(x)=0, s(x)=x^{-1 / 2}$, and $s(x)=\log x$. These integrals were also obtained analyticaliy and the relative error of the quadratures was computed. The numerical integrations were computed for various orders of quadrature and various numbers of nodes. Minimum sampling was taken to be two points per period of the cosine (i.e., $200 / \pi \approx 63.7$ quadrature nodes). The accuracies are then compared for various degrees of oversampling. The quadrature errors are listed in Tables 2 through 4 and plotted, as a function of oversampling factor, in Figure 1. We note that the graphs

TABLE 2. Relative errors in the computation of the integral in (94), for the regular case $s(x)=0$. Quadrature rules with convergence of order $2,4,8,16$, and 32 were used with various numbers $m=n+2 j$ of nodes. Here $f=m /(200 / \pi)$ is the oversampling factor.

\begin{tabular}{|rc|ccccc|}
\hline \multicolumn{1}{|c|}{$m$} & $f$ & 2 & 4 & 8 & 16 & 32 \\
\hline 70 & 1.10 & $0.622 \mathrm{D}+00$ & $0.114 \mathrm{D}-01$ & $0.382 \mathrm{D}-02$ & $0.170 \mathrm{D}-05$ & $0.234 \mathrm{D}-10$ \\
80 & 1.26 & $0.488 \mathrm{D}+00$ & $0.938 \mathrm{D}-02$ & $0.184 \mathrm{D}-02$ & $0.354 \mathrm{D}-06$ & $0.115 \mathrm{D}-11$ \\
90 & 1.41 & $0.391 \mathrm{D}+00$ & $0.744 \mathrm{D}-02$ & $0.934 \mathrm{D}-03$ & $0.841 \mathrm{D}-07$ & $0.720 \mathrm{D}-13$ \\
100 & 1.57 & $0.321 \mathrm{D}+00$ & $0.584 \mathrm{D}-02$ & $0.498 \mathrm{D}-03$ & $0.223 \mathrm{D}-07$ & $0.192 \mathrm{D}-14$ \\
115 & 1.81 & $0.246 \mathrm{D}+00$ & $0.408 \mathrm{D}-02$ & $0.211 \mathrm{D}-03$ & $0.365 \mathrm{D}-08$ & $0.331 \mathrm{D}-14$ \\
130 & 2.04 & $0.194 \mathrm{D}+00$ & $0.289 \mathrm{D}-02$ & $0.964 \mathrm{D}-04$ & $0.715 \mathrm{D}-09$ & $0.331 \mathrm{D}-14$ \\
145 & 2.28 & $0.157 \mathrm{D}+00$ & $0.209 \mathrm{D}-02$ & $0.472 \mathrm{D}-04$ & $0.162 \mathrm{D}-09$ & $0.471 \mathrm{D}-14$ \\
160 & 2.51 & $0.129 \mathrm{D}+00$ & $0.154 \mathrm{D}-02$ & $0.245 \mathrm{D}-04$ & $0.415 \mathrm{D}-10$ & $0.262 \mathrm{D}-14$ \\
180 & 2.83 & $0.102 \mathrm{D}+00$ & $0.106 \mathrm{D}-02$ & $0.110 \mathrm{D}-04$ & $0.794 \mathrm{D}-11$ & $0.471 \mathrm{D}-14$ \\
200 & 3.14 & $0.832 \mathrm{D}-01$ & $0.747 \mathrm{D}-03$ & $0.531 \mathrm{D}-05$ & $0.177 \mathrm{D}-11$ & $0.331 \mathrm{D}-14$ \\
230 & 3.61 & $0.631 \mathrm{D}-01$ & $0.465 \mathrm{D}-03$ & $0.199 \mathrm{D}-05$ & $0.235 \mathrm{D}-12$ & $0.523 \mathrm{D}-15$ \\
260 & 4.08 & $0.495 \mathrm{D}-01$ & $0.303 \mathrm{D}-03$ & $0.828 \mathrm{D}-06$ & $0.375 \mathrm{D}-13$ & $0.384 \mathrm{D}-14$ \\
\hline
\end{tabular}

TABLE 3. Relative errors for the singular case $s(x)=x^{-1 / 2}$, for various numbers $m=n+j+k$ of nodes and orders of convergence.

\begin{tabular}{|rc|cccc|}
\hline \multicolumn{1}{|c|}{$m$} & $f$ & 2 & 4 & 8 & 16 \\
\hline 70 & 1.10 & $0.692 \mathrm{D}-01$ & $0.519 \mathrm{D}-01$ & $0.850 \mathrm{D}-02$ & $0.163 \mathrm{D}-03$ \\
80 & 1.26 & $0.925 \mathrm{D}-01$ & $0.258 \mathrm{D}-01$ & $0.260 \mathrm{D}-02$ & $0.578 \mathrm{D}-05$ \\
90 & 1.41 & $0.921 \mathrm{D}-01$ & $0.133 \mathrm{D}-01$ & $0.698 \mathrm{D}-03$ & $0.667 \mathrm{D}-06$ \\
100 & 1.57 & $0.838 \mathrm{D}-01$ & $0.717 \mathrm{D}-02$ & $0.146 \mathrm{D}-03$ & $0.277 \mathrm{D}-06$ \\
115 & 1.81 & $0.686 \mathrm{D}-01$ & $0.307 \mathrm{D}-02$ & $0.201 \mathrm{D}-04$ & $0.360 \mathrm{D}-07$ \\
130 & 2.04 & $0.550 \mathrm{D}-01$ & $0.144 \mathrm{D}-02$ & $0.269 \mathrm{D}-04$ & $0.437 \mathrm{D}-08$ \\
145 & 2.28 & $0.441 \mathrm{D}-01$ & $0.724 \mathrm{D}-03$ & $0.171 \mathrm{D}-04$ & $0.557 \mathrm{D}-09$ \\
160 & 2.51 & $0.357 \mathrm{D}-01$ & $0.389 \mathrm{D}-03$ & $0.964 \mathrm{D}-05$ & $0.733 \mathrm{D}-10$ \\
180 & 2.83 & $0.273 \mathrm{D}-01$ & $0.186 \mathrm{D}-03$ & $0.440 \mathrm{D}-05$ & $0.408 \mathrm{D}-11$ \\
200 & 3.14 & $0.212 \mathrm{D}-01$ & $0.976 \mathrm{D}-04$ & $0.207 \mathrm{D}-05$ & $0.218 \mathrm{D}-12$ \\
230 & 3.61 & $0.151 \mathrm{D}-01$ & $0.427 \mathrm{D}-04$ & $0.724 \mathrm{D}-06$ & $0.130 \mathrm{D}-12$ \\
260 & 4.08 & $0.110 \mathrm{D}-01$ & $0.215 \mathrm{D}-04$ & $0.280 \mathrm{D}-06$ & $0.201 \mathrm{D}-13$ \\
\hline
\end{tabular}


TABLE 4. Relative errors for the singular case $s(x)=\log x$. Here the error is of order $O\left(h^{l} \log h\right)$, where $l$ is shown.

\begin{tabular}{|rc|cccc|}
\hline \multicolumn{1}{|c|}{$m$} & $f$ & 2 & 4 & 8 & 16 \\
\hline 70 & 1.10 & $0.369 \mathrm{D}+00$ & $0.217 \mathrm{D}-01$ & $0.354 \mathrm{D}-01$ & $0.243 \mathrm{D}-03$ \\
80 & 1.26 & $0.271 \mathrm{D}+00$ & $0.238 \mathrm{D}-02$ & $0.328 \mathrm{D}-02$ & $0.487 \mathrm{D}-04$ \\
90 & 1.41 & $0.206 \mathrm{D}+00$ & $0.765 \mathrm{D}-02$ & $0.707 \mathrm{D}-03$ & $0.394 \mathrm{D}-05$ \\
100 & 1.57 & $0.162 \mathrm{D}+00$ & $0.768 \mathrm{D}-02$ & $0.687 \mathrm{D}-03$ & $0.121 \mathrm{D}-05$ \\
115 & 1.81 & $0.117 \mathrm{D}+00$ & $0.576 \mathrm{D}-02$ & $0.291 \mathrm{D}-03$ & $0.886 \mathrm{D}-07$ \\
130 & 2.04 & $0.882 \mathrm{D}-01$ & $0.398 \mathrm{D}-02$ & $0.120 \mathrm{D}-03$ & $0.903 \mathrm{D}-08$ \\
145 & 2.28 & $0.687 \mathrm{D}-01$ & $0.272 \mathrm{D}-02$ & $0.548 \mathrm{D}-04$ & $0.123 \mathrm{D}-08$ \\
160 & 2.51 & $0.549 \mathrm{D}-01$ & $0.188 \mathrm{D}-02$ & $0.272 \mathrm{D}-04$ & $0.177 \mathrm{D}-09$ \\
180 & 2.83 & $0.421 \mathrm{D}-01$ & $0.119 \mathrm{D}-02$ & $0.118 \mathrm{D}-04$ & $0.965 \mathrm{D}-11$ \\
200 & 3.14 & $0.332 \mathrm{D}-01$ & $0.774 \mathrm{D}-03$ & $0.550 \mathrm{D}-05$ & $0.956 \mathrm{D}-12$ \\
230 & 3.61 & $0.243 \mathrm{D}-01$ & $0.433 \mathrm{D}-03$ & $0.196 \mathrm{D}-05$ & $0.398 \mathrm{D}-12$ \\
260 & 4.08 & $0.185 \mathrm{D}-01$ & $0.258 \mathrm{D}-03$ & $0.778 \mathrm{D}-06$ & $0.106 \mathrm{D}-12$ \\
\hline
\end{tabular}
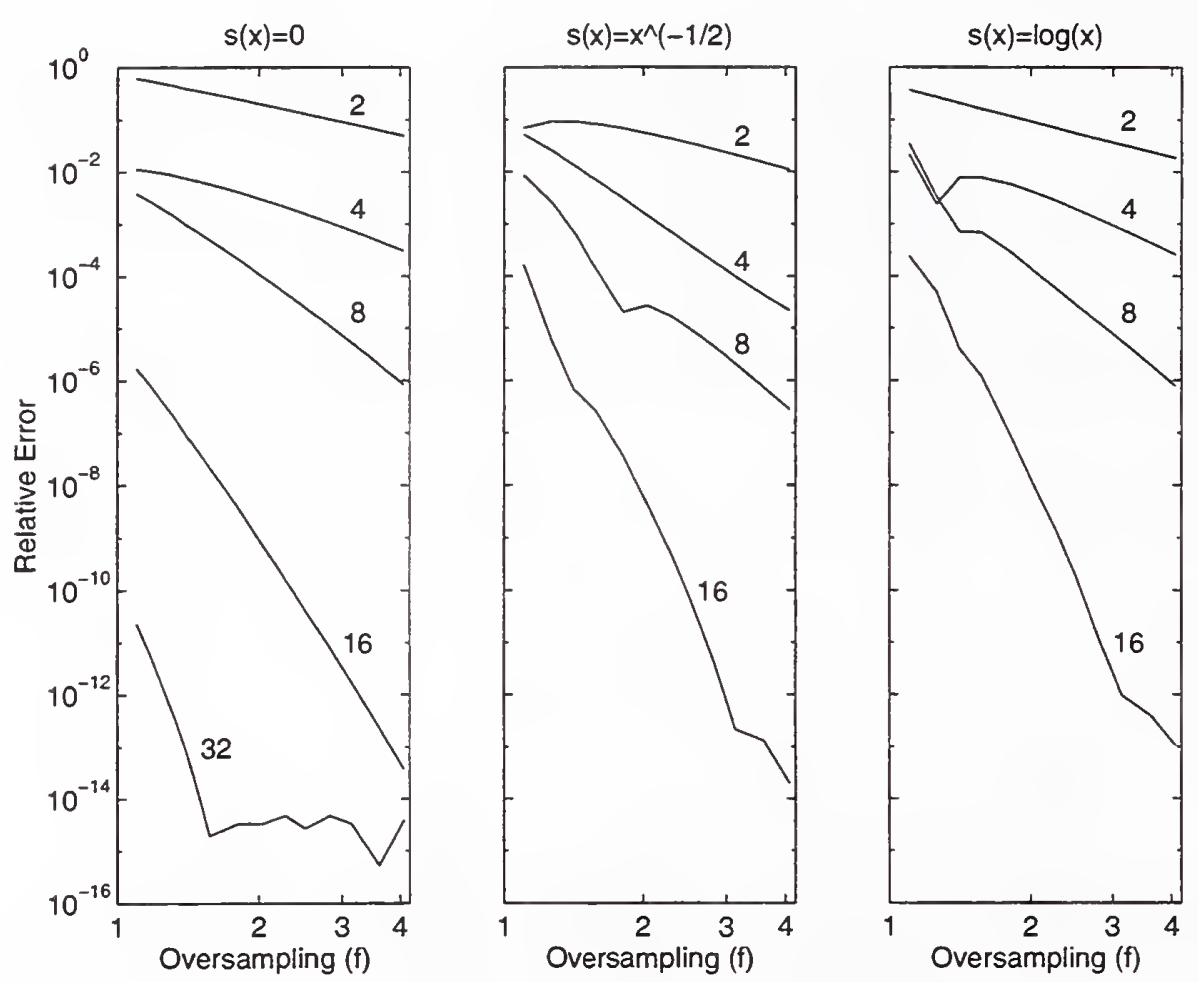

FIGURE 1. The relative errors of the quadratures, shown in Tables 2 through 4 , are plotted using logarithmic scaling on both axes.

are nearly straight lines (until the limit of machine precision is reached), as predicted from the theoretical convergence rates. We remark also that excellent accuracy is attained for even quite modest oversampling when quadratures with high-order convergence are employed. For problems where the 
TABLE 5. Relative errors in the computation of the integral in (95), for $\xi=1$. Quadrature rules defined in Corollary 3.10 with $j=1,2,4,8$, and 16 were used with various numbers $m$ of nodes.

\begin{tabular}{|r|ccccc|}
\hline \multicolumn{1}{c|}{$m$} & 1 & 2 & 4 & 8 & 16 \\
\hline 70 & $0.999 \mathrm{D}+00$ & $0.400 \mathrm{D}+00$ & $0.305 \mathrm{D}+00$ & $0.180 \mathrm{D}+00$ & $0.104 \mathrm{D}-01$ \\
80 & $0.304 \mathrm{D}+00$ & $0.200 \mathrm{D}-01$ & $0.247 \mathrm{D}-01$ & $0.238 \mathrm{D}-02$ & $0.474 \mathrm{D}-03$ \\
90 & $0.113 \mathrm{D}+00$ & $0.217 \mathrm{D}-01$ & $0.136 \mathrm{D}-02$ & $0.383 \mathrm{D}-03$ & $0.866 \mathrm{D}-05$ \\
100 & $0.273 \mathrm{D}-01$ & $0.137 \mathrm{D}-01$ & $0.137 \mathrm{D}-02$ & $0.440 \mathrm{D}-04$ & $0.900 \mathrm{D}-06$ \\
115 & $0.210 \mathrm{D}-01$ & $0.247 \mathrm{D}-02$ & $0.137 \mathrm{D}-03$ & $0.573 \mathrm{D}-05$ & $0.331 \mathrm{D}-07$ \\
130 & $0.228 \mathrm{D}-01$ & $0.107 \mathrm{D}-02$ & $0.632 \mathrm{D}-04$ & $0.423 \mathrm{D}-06$ & $0.107 \mathrm{D}-09$ \\
145 & $0.118 \mathrm{D}-01$ & $0.115 \mathrm{D}-02$ & $0.305 \mathrm{D}-04$ & $0.196 \mathrm{D}-06$ & $0.490 \mathrm{D}-10$ \\
160 & $0.212 \mathrm{D}-02$ & $0.521 \mathrm{D}-03$ & $0.307 \mathrm{D}-05$ & $0.430 \mathrm{D}-07$ & $0.216 \mathrm{D}-09$ \\
180 & $0.625 \mathrm{D}-02$ & $0.824 \mathrm{D}-04$ & $0.451 \mathrm{D}-05$ & $0.101 \mathrm{D}-07$ & $0.166 \mathrm{D}-11$ \\
200 & $0.558 \mathrm{D}-02$ & $0.206 \mathrm{D}-03$ & $0.184 \mathrm{D}-05$ & $0.291 \mathrm{D}-08$ & $0.867 \mathrm{D}-12$ \\
230 & $0.863 \mathrm{D}-03$ & $0.676 \mathrm{D}-04$ & $0.463 \mathrm{D}-06$ & $0.626 \mathrm{D}-09$ & $0.586 \mathrm{D}-13$ \\
260 & $0.266 \mathrm{D}-02$ & $0.433 \mathrm{D}-04$ & $0.291 \mathrm{D}-06$ & $0.596 \mathrm{D}-10$ & $0.346 \mathrm{D}-14$ \\
\hline
\end{tabular}

number of quadrature nodes is the major cost factor, therefore, one may benefit by using the high-order quadratures even for modest accuracy requirements.

We test the quadratures for improper integrals by numerically computing for $\xi=1$ the integral

$$
\int_{-\infty}^{\infty} e^{-i x \xi} \sum_{r=-10}^{10} \frac{r+1}{x+r+i} d x=-2 \pi i H(\xi) \sum_{r=-10}^{10}(r+1) e^{i r \xi-\xi},
$$

where $H$ is the Heaviside step function. The integrand is oscillatory and decays like $x^{-1}$ as $x \rightarrow \pm \infty$. The quadratures defined in Corollary 3.10 are employed, for which the integral is split into a regular integral on a finite interval, chosen here to be $[-5 \sqrt{m} / 4,5 \sqrt{m} / 4]$, where $m$ is the total number of quadrature nodes, and two improper integrals in the imaginary direction, using Laguerre quadratures. The quadrature errors are shown in Table 5.

\section{APPLICATIONS AND SUMMARY}

The chief motivation for the hybrid Gauss-trapezoidal quadrature rules is the accurate computation of integral operators. We define an integral operator $A$ by the formula

$$
(A f)(x)=\int_{\Gamma} K(x, y) f(y) d y,
$$

where $\Gamma$ is a regular, simple closed curve in the complex plane, the function $f$ is regular on $\Gamma$, and the kernel $K: \mathbb{C} \times \mathbb{C} \rightarrow \mathbb{C}$ is a regular function of its arguments, except where they coincide; we assume

$$
K(x, y)=\phi(x, y) s(|x-y|)+\psi(x, y),
$$

with $\phi$ and $\psi$ regular on $\Gamma \times \Gamma$ and $s$ regular on $(0, \infty)$, with an integrable singularity at 0 . A large variety of problems of classical physics can be formulated as integral equations that involve such operators. When the operator occurs in an integral equation

$$
f(x)+(A f)(x)=g(x), \quad x \in \Gamma,
$$


some choice of discretization must be used to reduce the problem to a finite-dimensional one for numerical solution. In the Nyström method the integrals are replaced by quadratures to yield the finite system of equations

$$
f\left(x_{i}\right)+\sum_{j=1}^{m} w_{i j} f\left(x_{j}\right)=g\left(x_{i}\right), \quad i=1, \ldots, m .
$$

This linear system can be solved for $f\left(x_{1}\right), \ldots, f\left(x_{m}\right)$ by a variety of techniques. The particular choice of $x_{i}$ and $w_{i j}$ for $i, j=1, \ldots, m$ determines the order of convergence (and therefore efficiency) of the method.

For a curve parametrization $v:[0,1] \rightarrow \Gamma$, such as scaled arc length, the operator $A$ becomes

$$
(A f)(v(t))=\int_{0}^{1} K(v(t), v(\tau)) f(v(\tau)) v^{\prime}(\tau) d \tau .
$$

It is convenient to use a uniform discretization $1 / m, 2 / m, \ldots, 1$ in $t$ and $\tau$, so $x_{i}=v(i / m), i=$ $1, \ldots, m$. How then is $w_{i j}$ determined? We assume for the moment that $f$ is available at locations other than $x_{1}, \ldots, x_{m}$. Continuing $v$ periodically with period 1 , and using the Gauss-trapezoidal quadratures, we obtain

$$
\begin{aligned}
(A f)(v(i / m)) & =\int_{i / m}^{1+i / m} K(v(i / m), v(\tau)) f\left(v(\tau) v^{\prime}(\tau) d \tau\right. \\
\approx & \frac{1}{m} \sum_{k=1}^{j} u_{k} \sigma_{i / m}\left(v_{k} / m\right)+\frac{1}{m} \sum_{k=0}^{n-1} \sigma_{i / m}(a / m+k / m)+\frac{1}{m} \sum_{k=1}^{j} u_{k} \sigma_{i / m}\left(1-v_{k} / m\right)
\end{aligned}
$$

for $i=1, \ldots, m$, where $\sigma_{\alpha}:[0,1] \rightarrow \mathbb{C}$ is defined by the formula

$$
\sigma_{\alpha}(\tau)=K(\nu(\alpha), v(\alpha+\tau)) f(\alpha+\tau) v^{\prime}(\alpha+\tau)
$$

and $m=n+2 a-1$ and $u_{1}, \ldots, u_{j}, v_{1}, \ldots, v_{j}$ are determined for the singularity $s$ of $K$. Provided that the periodic continuation of $v$ is sufficiently regular, the quadrature will converge to the integral with order greater than $j$ as $m \rightarrow \infty$, for $i=1, \ldots, m$. We relax the restriction that $f$ be available outside $x_{1}, \ldots, x_{m}$ by using local Lagrange interpolation of order $j+1$ for equispaced nodes,

$$
f(v(\tau)) \approx \sum_{r=0}^{j} f(v(i / m+r / m)) l_{r}(m \tau-i),
$$

where $i=\lfloor m \tau-(j-1) / 2\rfloor$ and

$$
l_{r}(x)=\prod_{s=0, s \neq r}^{j} \frac{x-s}{r-s}, \quad r=0, \ldots, j .
$$

Now $w_{i j}$ for $i, j=1, \ldots, m$ is determined by combining (97) through (101). The computation of all $m^{2}$ coefficients requires $m(m+2 j-2 a+1)$ evaluations of the kernel $K$ and therefore order $O\left(\mathrm{~m}^{2}\right)$ operations. This cost can often be substantially reduced using techniques that exploit kernel smoothness (see, for example, [17], [18]).

A slightly different application of the quadratures is the computation of Fourier transforms of functions that fail to satisfy the assumptions usually made when using the discrete Fourier transform. In particular, if a function decays slowly for large argument or is compactly supported and singular at the ends of the support interval, these quadratures can be used to compute its Fourier transform. One 
example of such a function is that in (95). Since most of the nodes in these quadratures are equispaced, with function values given equal weight, the fast Fourier transform can be used to do the bulk of the computations; the overall complexity is $O(n \log n)$, where $n$ is the number of Fourier coefficients to be computed.

Other applications may include the representation of functions for solving ordinary or partial differential equations, when high-order methods are required. In addition, an extension of these quadratures to integrals on surfaces is under study.

To summarize, the characteristics of the hybrid Gauss-trapezoidal quadrature rules include

- Arbitrary order convergence for regular functions or functions with known singularities of power or logarithmic type,

- Positive weights,

- Most nodes equispaced and most weights constant, and

- Invariant nodes and weights (aside from scaling) as the problem size increases.

The primary disadvantage of the quadrature rules, shared with other Gaussian quadratures but exacerbated here by poor conditioning, is that the computation of the nodes and weights is not trivial. Nevertheless, tabulation of nodes and weights for a given order of convergence allows this issue to be avoided in the construction of high-order, general purpose quadrature routines.

Acknowledgments. The author would like to thank Gregory Beylkin, Vladimir Rokhlin, and Ronald Wittmann for helpful discussions and encouragement. This research was supported in part by Defense Advanced Research Projects Agency grant 9760400 and in part by the University of Colorado Program in Applied Mathematics, Boulder, where the author was welcomed during the government furloughs of 1995-1996.

\section{REFERENCES}

[1] V. Rokhlin. End-point corrected trapezoidal quadrature rules for singular functions. Computers and Mathematics with Applications, 20:51-62, 1990.

[2] H. P. Starr. On the Numerical Solution of One-Dimensional Integral and Differential Equations. PhD thesis, Yale University, December, 1991.

[3] B. Alpert. High-order quadratures for integral operators with singular kernels. Journal of Computational and Applied Mathematics, 60:367-378, 1995.

[4] Rainer Kress. A Nyström method for boundary integral equations in domains with corners. Numerische Mathematik, 58:145-161, 1990.

[5] S. Kapur and V. Rokhlin. High-order corrected trapezoidal rules for singular functions. SIAM Journal on Numerical Analysis, to appear, 1996.

[6] J. Ma, V. Rokhlin, and S. Wandzura. Generalized Gaussian quadrature rules for systems of arbitrary functions. SIAM Journal on Numerical Analysis, 33:971-996, 1996.

[7] M. Abramowitz and I. A. Stegun, editors. Handbook of Mathematical Functions (10th printing). National Bureau of Standards, Applied Mathematics Series 55, 1972.

[8] A. Erdélyi, editor. Higher Transcendental Functions, volume 1. Krieger Publishing Company, Malabar, Florida, 1985. Reprint of McGraw-Hill edition, 1953.

[9] C. M. Bender and S. A. Orszag. Advanced Mathematical Methods for Scientists and Engineers. McGraw Hill, New York, 1978.

[10] J. Stoer and R. Bulirsch. Introduction to Numerical Analysis. Springer Verlag, 1980.

[11] G. Szegö. Orthogonal Polynomials. American Mathematical Society, 1939.

[12] S. Karlin and W. Studden. Tchebycheff Systems with Applications in Analysis and Statistics. Wiley and Sons, New York, 1966.

[13] Peter Borwein and Tamás Erdélyi. Polynomials and Polynomial Inequalities. Springer-Verlag, New York, 1995. 
[14] C. Batut, D. Bernardi, H. Cohen, and M. Olivier. User's Guide to PAR1-GP. Technical report, Laboratoire A2X, Université Bordeaux I (ftp: megrez.math.u-bordeaux.fr), 1995.

[15] Walter Gautschi. On the construction of Gaussian quadrature rules from modified moments. Mathematics of Computation, 24:245-260, 1970.

[16] G. H. Golub and J. H. Welsch. Calculation of Gaussian quadrature rules. Mathematics of Computation, 23:221-230, 1969.

[17] B. Alpert, G. Beylkin, R. Coifman, and V. Rokhlin. Wavelet-like bases for the fast solution of second-kind integral equations. SIAM Journal on Scientific Computing, 14:159-184, 1993.

[18] G. Beylkin, R. Coifman, and V. Rokhlin. Fast wavelet transforms and numerical algorithms I. Communications on Pure and Applied Mathematics, XL1V:141-183, 1991. 
APPENDiX. TABLES of QUADRATURE NodEs AND WeightS

TABLE 6. The nodes and weights for the quadrature rule $T_{n}^{j a}(f)=$ $h \sum_{i=1}^{j} w_{i} f\left(x_{i} h\right)+h \sum_{i=0}^{n-1} f(a h+i h)+h \sum_{i=1}^{j} w_{i} f\left(1-x_{i} h\right)$, with $h=(n+2 a-1)^{-1}$, for several choices of $j$ and corresponding minimum integer $a$. For $f$ a regular function, $T_{n}^{j a}(f)$ converges to $\int_{0}^{1} f(x) d x$ as $n \rightarrow \infty$ with convergence of order $O$.

\begin{tabular}{|c|c|c|c|}
\hline$O$ & & $x_{i}$ & $w_{i}$ \\
\hline 3 & 1 & $1.6666666666666667 \mathrm{D}-01$ & $5.000000000000000 \mathrm{D}-01$ \\
\hline \multirow[t]{2}{*}{4} & 2 & $2.000000000000000 \mathrm{D}-01$ & $5.2083333333333333 \mathrm{D}-01$ \\
\hline & & $1.000000000000000 \mathrm{D}+00$ & $9.791666666666667 \mathrm{D}-01$ \\
\hline \multirow[t]{2}{*}{5} & 2 & $2.245784979812614 \mathrm{D}-01$ & $5.540781643606372 \mathrm{D}-01$ \\
\hline & & $1.013719374359164 \mathrm{D}+00$ & $9.459218356393628 \mathrm{D}-01$ \\
\hline \multirow[t]{3}{*}{6} & 3 & $2.250991042610971 \mathrm{D}-01$ & $5.549724327164180 \mathrm{D}-01$ \\
\hline & & $1.014269060987992 \mathrm{D}+00$ & $9.451317411845473 \mathrm{D}-01$ \\
\hline & & $2.000000000000000 \mathrm{D}+00$ & $9.998958260990347 \mathrm{D}-01$ \\
\hline \multirow[t]{3}{*}{7} & 3 & $2.180540672543505 \mathrm{D}-01$ & $5.408088967208193 \mathrm{D}-01$ \\
\hline & & $1.001181873031216 \mathrm{D}+00$ & $9.516615045823566 \mathrm{D}-01$ \\
\hline & & $1.997580526418033 \mathrm{D}+00$ & $1.007529598696824 \mathrm{D}+00$ \\
\hline \multirow[t]{4}{*}{8} & 4 & $2.087647422032129 \mathrm{D}-01$ & $5.207988277246498 \mathrm{D}-01$ \\
\hline & & $9.786087373714483 \mathrm{D}-01$ & $9.535038018555888 \mathrm{D}-01$ \\
\hline & & $1.989541386579751 \mathrm{D}+00$ & $1.024871626402471 \mathrm{D}+00$ \\
\hline & & $3.000000000000000 \mathrm{D}+00$ & $1.000825744017291 \mathrm{D}+00$ \\
\hline \multirow[t]{6}{*}{12} & 5 & $7.023955461621939 \mathrm{D}-02$ & $1.922315977843698 \bar{D}-01$ \\
\hline & & $4.312297857227970 \mathrm{D}-01$ & $5.348399530514687 \mathrm{D}-01$ \\
\hline & & $1.117752734518115 \mathrm{D}+00$ & $8.170209442488760 \mathrm{D}-01$ \\
\hline & & $2.017343724572518 \mathrm{D}+00$ & $9.592111521445966 \mathrm{D}-01$ \\
\hline & & $3.000837842847590 \mathrm{D}+00$ & $9.967143408044999 \mathrm{D}-01$ \\
\hline & & $4.000000000000000 D+00$ & $9.999820119661890 \mathrm{D}-01$ \\
\hline \multirow[t]{8}{*}{16} & 7 & $9.919337841451028 \mathrm{D}-02$ & $2.528198928766921 \mathrm{D}-01$ \\
\hline & & $5.076592669645529 \mathrm{D}-01$ & $5.550158230159486 \mathrm{D}-01$ \\
\hline & & $1.184972925827278 \mathrm{D}+00$ & $7.852321453615224 \mathrm{D}-01$ \\
\hline & & $2.047493467134072 \mathrm{D}+00$ & $9.245915673876714 \mathrm{D}-01$ \\
\hline & & $3.007168911869310 \mathrm{D}+00$ & $9.839350200445296 \mathrm{D}-01$ \\
\hline & & $4.000474996776184 \mathrm{D}+00$ & $9.984463448413151 \mathrm{D}-01$ \\
\hline & & $5.000007879022339 \mathrm{D}+00$ & $9.999592378464547 \mathrm{D}-01$ \\
\hline & & $6.000000000000000 \mathrm{D}+00$ & $9.999999686258662 \mathrm{D}-01$ \\
\hline \multirow[t]{10}{*}{20} & 9 & $9.209200446233291 \mathrm{D}-02$ & $2.351836144643984 \mathrm{D}-01$ \\
\hline & & $4.752021947758861 \mathrm{D}-01$ & $5.248820509085946 \mathrm{D}-01$ \\
\hline & & $1.124687945844539 \mathrm{D}+00$ & $7.634026409869887 \mathrm{D}-01$ \\
\hline & & $1.977387385642367 \mathrm{D}+00$ & $9.284711336658351 \mathrm{D}-01$ \\
\hline & & $2.953848957822108 \mathrm{D}+00$ & $1.010969886587741 \mathrm{D}+00$ \\
\hline & & $3.976136786048776 \mathrm{D}+00$ & $1.024959725311073 \mathrm{D}+00$ \\
\hline & & $4.994354281979877 \mathrm{D}+00$ & $1.010517534639652 \mathrm{D}+00$ \\
\hline & & $5.999469539335291 \mathrm{D}+00$ & $1.001551595797932 \mathrm{D}+00$ \\
\hline & & $6.999986704874333 \mathrm{D}+00$ & $1.000061681794188 \mathrm{D}+00$ \\
\hline & & $8.000000000000000 D+00$ & $1.000000135843597 \mathrm{D}+00$ \\
\hline
\end{tabular}


TABLE 6. (CONTINUED)

\begin{tabular}{|c|c|c|c|}
\hline 0 & $a$ & $x_{i}$ & $w_{i}$ \\
\hline \multirow[t]{12}{*}{24} & 10 & $6.001064731474805 \mathrm{D}-02$ & $1.538932104518340 \mathrm{D}-01$ \\
\hline & & $3.149685016229433 \mathrm{D}-01$ & $3.551058128559424 \mathrm{D}-01$ \\
\hline & & $7.664508240518316 \mathrm{D}-01$ & $5.449200036280007 \mathrm{D}-01$ \\
\hline & & $1.396685781342510 \mathrm{D}+00$ & 7.1040784977 15549D-01 \\
\hline & & $2.175195903206602 \mathrm{D}+00$ & $8.398780940253654 \mathrm{D}-01$ \\
\hline & & $3.062320575880355 \mathrm{D}+00$ & $9.272767950890611 \mathrm{D}-01$ \\
\hline & & $4.016440988792476 \mathrm{D}+00$ & $9.750605697371132 \mathrm{D}-01$ \\
\hline & & $5.002872064275734 \mathrm{D}+00$ & $9.942629650823470 \mathrm{D}-01$ \\
\hline & & $6.000285453310164 \mathrm{D}+00$ & $9.992421778421898 \mathrm{D}-01$ \\
\hline & & $7.000012964962529 \mathrm{D}+00$ & $9.999534370786161 \mathrm{D}-01$ \\
\hline & & $8.000000175554469 \mathrm{D}+00$ & $9.999990854912925 \mathrm{D}-01$ \\
\hline & & $9.000000000000000 \mathrm{D}+00$ & $9.999999989466828 \mathrm{D}-01$ \\
\hline \multirow[t]{14}{*}{28} & 12 & $6.234360533194102 \mathrm{D}-02$ & $1.595975279734157 \mathrm{D}-01$ \\
\hline & & $3.250286721702614 \mathrm{D}-01$ & $3.637046028193864 \mathrm{D}-01$ \\
\hline & & $7.837350794282182 \mathrm{D}-01$ & $5.498753177297441 \mathrm{D}-01$ \\
\hline & & $1.415673112616924 \mathrm{D}+00$ & 7.087986792086 \\
\hline & & $3 \mathrm{D}+00$ & 8.335 \\
\hline & & 3.07 & $8 \mathrm{D}-01$ \\
\hline & & $7 \mathrm{D}+00$ & $90 \mathrm{D}-01$ \\
\hline & & $97 \mathrm{D}+00$ & $9.933296578555239 \mathrm{D}-01$ \\
\hline & & $29741810400 D+00$ & $9.994759087910050 \mathrm{D}-01$ \\
\hline & & $6.999904720846024 \mathrm{D}+00$ & $1.000133030254421 \mathrm{D}+00$ \\
\hline & & $7.999986894843540 \mathrm{D}+00$ & $1.000032915011460 \mathrm{D}+00$ \\
\hline & & $8.999999373380393 \mathrm{D}+00$ & $1.000002261653775 \mathrm{D}+00$ \\
\hline & & $9.999999992002911 \mathrm{D}+00$ & $1.000000042393520 \mathrm{D}+00$ \\
\hline & & $00 \mathrm{D}+01$ & $42872 D+00$ \\
\hline \multirow[t]{16}{*}{32} & 14 & $9 \mathrm{D}-02$ & 79D-01 \\
\hline & & 3.082757062227 & $169090 \mathrm{D}-01$ \\
\hline & & $7.463707253079130 \mathrm{D}-01$ & $146873 D-01$ \\
\hline & & $1.355993726494664 \mathrm{D}+00$ & $6.878444094543021 \mathrm{D}-01$ \\
\hline & & $2.112943217346336 \mathrm{D}+00$ & $8.210319140034114 \mathrm{D}-01$ \\
\hline & & $2.987241496545946 \mathrm{D}+00$ & $9.218382875515803 \mathrm{D}-01$ \\
\hline & & $3.944798920961176 \mathrm{D}+00$ & $9.873027487553060 \mathrm{D}-01$ \\
\hline & & $4.950269202842798 \mathrm{D}+00$ & $1.018251913441155 \mathrm{D}+00$ \\
\hline & & $06 \mathrm{D}+00$ & 1.02193343 \\
\hline & & 6.989783558137 & 1.01256798 \\
\hline & & 7.997673019512 & 1.004052289 \\
\hline & & $8.999694932747039 D+00$ & $1.000713413344501 \mathrm{D}+00$ \\
\hline & & $9.999979225211805 \mathrm{D}+00$ & $1.000063618302950 \mathrm{D}+00$ \\
\hline & & $1.099999938266130 \mathrm{D}+01$ & $1.000002486385216 \mathrm{D}+00$ \\
\hline & & $1.199999999462073 \mathrm{D}+01$ & $1.000000030404477 \mathrm{D}+00$ \\
\hline & & $1.300000000000000 \mathrm{D}+01$ & $1.000000000020760 \mathrm{D}+00$ \\
\hline
\end{tabular}


TABLE 7. The nodes $v_{1}, \ldots, v_{j}$ and weights $u_{1}, \ldots, u_{j}$ for the quadrature rule $S_{n}^{j k a b}(g)=h \sum_{i=1}^{j} u_{i} g\left(v_{i} h\right)+h \sum_{i=0}^{n-1} g(a h+i h)+h \sum_{i=1}^{k} w_{i} g\left(1-x_{i} h\right)$, with $h=(n+a+b-1)^{-1}$, for $g(x)=x^{-1 / 2} \phi(x)+\psi(x)$, with $\phi$ and $\psi$ regular functions. The nodes $x_{1}, \ldots, x_{k}$ and weights $w_{1}, \ldots, w_{k}$ are found in Table 6.

\begin{tabular}{|c|c|c|c|}
\hline$O$ & $a$ & $v_{i}$ & $u_{i}$ \\
\hline 1.5 & 1 & $1.172258571393266 \mathrm{D}-01$ & $5.000000000000000 \mathrm{D}-01$ \\
\hline \multirow[t]{2}{*}{2.0} & 2 & $9.252112715421378 \mathrm{D}-02$ & $4.198079625266162 \mathrm{D}-01$ \\
\hline & & $1.000000000000000 \mathrm{D}-00$ & $1.080192037473384 \mathrm{D}+00$ \\
\hline \multirow[t]{2}{*}{2.5} & 2 & $6.023873796408450 \mathrm{D}-02$ & $2.858439990420468 \mathrm{D}-01$ \\
\hline & & $8.780704050676215 \mathrm{D}-01$ & $1.214156000957953 \mathrm{D}+00$ \\
\hline \multirow[t]{3}{*}{3.0} & 2 & $7.262978413470474 \mathrm{D}-03$ & $3.907638767531813 \mathrm{D}-02$ \\
\hline & & $2.246325512521893 \mathrm{D}-01$ & $4.873484056646474 \mathrm{D}-01$ \\
\hline & & $1.000000000000000 \mathrm{D}+00$ & $9.735752066600344 \mathrm{D}-01$ \\
\hline \multirow[t]{3}{*}{3.5} & 2 & $1.282368909458828 \mathrm{D}-02$ & $6.363996663105925 \mathrm{D}-02$ \\
\hline & & $2.694286346792474 \mathrm{D}-01$ & $5.077434578043636 \mathrm{D}-01$ \\
\hline & & $1.018414523786358 \mathrm{D}+00$ & $9.286165755645772 \mathrm{D}-01$ \\
\hline \multirow[t]{4}{*}{4.0} & 3 & $1.189242434021285 \mathrm{D}-02$ & $5.927215035616424 \mathrm{D}-02$ \\
\hline & & $2.578220434738662 \mathrm{D}-01$ & $4.955981740306228 \mathrm{D}-01$ \\
\hline & & $1.007750064585281 \mathrm{D}+00$ & $9.427131290628058 \mathrm{D}-01$ \\
\hline & & $2.000000000000000 \mathrm{D}+00$ & $1.002416546550407 \mathrm{D}+00$ \\
\hline \multirow[t]{6}{*}{6.0} & 4 & $3.317925942699451 \mathrm{D}-03$ & $1.681780929883469 \mathrm{D}-02$ \\
\hline & & $8.283019705296352 \mathrm{D}-02$ & $1.755244404544475 \mathrm{D}-01$ \\
\hline & & $4.136094925726231 \mathrm{D}-01$ & $5.039350503858001 \mathrm{D}-01$ \\
\hline & & $1.088744373688402 \mathrm{D}+00$ & $8.266241339680867 \mathrm{D}-01$ \\
\hline & & $2.006482101852379 \mathrm{D}+00$ & $9.773065848981277 \mathrm{D}-01$ \\
\hline & & $3.000000000000000 \mathrm{D}+00$ & $9.997919809947032 \mathrm{D}-01$ \\
\hline \multirow[t]{8}{*}{8.0} & 5 & $1.214130606523435 \mathrm{D}-03$ & $6.199844884297793 \mathrm{D}-03$ \\
\hline & & $3.223952700027058 \mathrm{D}-02$ & $7.106286791720044 \mathrm{D}-02$ \\
\hline & & $1.790935383649920 \mathrm{D}-01$ & $2.408930104410471 \mathrm{D}-01$ \\
\hline & & $5.437663805244631 \mathrm{D}-01$ & $4.975929263668960 \mathrm{D}-01$ \\
\hline & & $1.176116628396759 \mathrm{D}+00$ & $7.592446540441226 \mathrm{D}-01$ \\
\hline & & $2.031848210716014 \mathrm{D}+00$ & $9.322446399614420 \mathrm{D}-01$ \\
\hline & & $3.001961225690812 D+00$ & $9.928171438160095 \mathrm{D}-01$ \\
\hline & & $4.000000000000000 \mathrm{D}+00$ & $9.999449125689846 \mathrm{D}-01$ \\
\hline \multirow[t]{10}{*}{10.0} & 6 & $1.745862989163252 \mathrm{D}-04$ & $1.016950985948944 \mathrm{D}-03$ \\
\hline & & $8.613670540457314 \mathrm{D}-03$ & $2.294670686517670 \mathrm{D}-02$ \\
\hline & & $6.733385088703690 \mathrm{D}-02$ & $1.076657968022888 \mathrm{D}-01$ \\
\hline & & $2.514488774733840 \mathrm{D}-01$ & $2.734577662465576 \mathrm{D}-01$ \\
\hline & & $6.341845573737690 \mathrm{D}-01$ & $4.978815591924992 \mathrm{D}-01$ \\
\hline & & $1.248404055083152 \mathrm{D}+00$ & $7.256208919565360 \mathrm{D} \rightarrow 01$ \\
\hline & & $2.065688031953401 \mathrm{D}+00$ & $8.952638690320078 D-01$ \\
\hline & & $3.009199358662542 \mathrm{D}+00$ & $9.778157465381624 \mathrm{D}-01$ \\
\hline & & $4.000416269690208 \mathrm{D}+00$ & $9.983390781399277 \mathrm{D}-01$ \\
\hline & & $5.000000000000000 \mathrm{D}+00$ & $9.999916342408948 \mathrm{D}-01$ \\
\hline
\end{tabular}


TABle 7. (CONTINUED)

\begin{tabular}{|c|c|c|c|}
\hline$O$ & $a$ & $v_{i}$ & $u_{i}$ \\
\hline \multirow[t]{12}{*}{12.0} & 8 & $5.710218427206990 \mathrm{D}-04$ & $2.921018926912141 \mathrm{D}-03$ \\
\hline & & 1.5404243511 15548D-02 & $3.431130611256885 \mathrm{D}-02$ \\
\hline & & $8.834248407196555 \mathrm{D}-02$ & $1.224669495638615 \mathrm{D}-01$ \\
\hline & & $2.824462054509770 \mathrm{D}-01$ & $2.761108242022520 \mathrm{D}-01$ \\
\hline & & $6.574869892305580 \mathrm{D}-01$ & $4.797809643010337 \mathrm{D}-01$ \\
\hline & & $1.246541060977993 \mathrm{D}+00$ & $6.966555677271379 \mathrm{D}-01$ \\
\hline & & $2.039218495130811 \mathrm{D}+00$ & $8.790077941972658 \mathrm{D}-01$ \\
\hline & & $2.979333487049800 \mathrm{D}+00$ & $9.868622449294327 \mathrm{D}-01$ \\
\hline & & 3.9857725953 93049D+00 & $1.015142389688201 \mathrm{D}+00$ \\
\hline & & $4.997240804311428 \mathrm{D}+00$ & $1.006209712632210 \mathrm{D}+00$ \\
\hline & & $5.999868793951190 \mathrm{D}+00$ & $1.000528829922287 \mathrm{D}+00$ \\
\hline & & $7.000000000000000 \mathrm{D}+00$ & $1.000002397796838 \mathrm{D}+00$ \\
\hline \multirow[t]{14}{*}{14.0} & 9 & $3.419821460249725 \mathrm{D}-04$ & $1.750957243202047 \mathrm{D}-03$ \\
\hline & & $9.296593430187960 \mathrm{D}-03$ & $2.080726584287380 \mathrm{D}-02$ \\
\hline & & $5.406214771755252 \mathrm{D}-02$ & $7.586830616433430 \mathrm{D}-02$ \\
\hline & & $1.763945096508648 \mathrm{D}-01$ & $1.766020526671851 \mathrm{D}-01$ \\
\hline & & $4.218486605653738 \mathrm{D}-01$ & $3.206624362072232 \mathrm{D}-01$ \\
\hline & & $8.274022895884040 \mathrm{D}-01$ & $4.934405290553812 \mathrm{D}-01$ \\
\hline & & $1.410287585637014 \mathrm{D}+00$ & $6.707497030698472 \mathrm{D}-01$ \\
\hline & & $2.160997505238153 \mathrm{D}+00$ & $8.244959025366557 \mathrm{D}-01$ \\
\hline & & $3.043504749358223 \mathrm{D}+00$ & $9.314646742162802 \mathrm{D}-01$ \\
\hline & & $4.005692579069439 \mathrm{D}+00$ & $9.845768443163154 \mathrm{D}-01$ \\
\hline & & $4.999732707905968 \mathrm{D}+00$ & $9.992852769154770 \mathrm{D}-01$ \\
\hline & & $5.999875191971098 \mathrm{D}+00$ & $1.000273112957723 \mathrm{D}+00$ \\
\hline & & $6.999994560568667 \mathrm{D}+00$ & $1.000022857402321 \mathrm{D}+00$ \\
\hline & & $8.000000000000000 \mathrm{D}+00$ & $1.000000081405180 \mathrm{D}+00$ \\
\hline \multirow[t]{16}{*}{16.0} & 10 & $2.158438988280793 \mathrm{D}-04$ & $1.105804873501181 \mathrm{D}-03$ \\
\hline & & $5.898432743709196 \mathrm{D}-03$ & $1.324499944707956 \mathrm{D}-02$ \\
\hline & & $3.462795956896131 \mathrm{D}-02$ & $4.899842307592144 \mathrm{D}-02$ \\
\hline & & $1.145586495070213 \mathrm{D}-01$ & $1.165326192868815 \mathrm{D}-01$ \\
\hline & & $2.790344218856415 \mathrm{D}-01$ & $2.178586693194957 \mathrm{D}-01$ \\
\hline & & $5.600113798653321 \mathrm{D}-01$ & $3.481766016945031 \mathrm{D}-01$ \\
\hline & & $9.814091242883119 \mathrm{D}-01$ & $4.964027915911545 \mathrm{D}-01$ \\
\hline & & $1.553594853974655 \mathrm{D}+00$ & $6.469026189623831 \mathrm{D}-01$ \\
\hline & & $2.270179114036658 \mathrm{D}+00$ & $7.823688971783889 \mathrm{D}-01$ \\
\hline & & 3.1082346017 15371D+00 & $8.877772445893361 \mathrm{D}-01$ \\
\hline & & $4.032930893996553 \mathrm{D}+00$ & $9.551665077035583 \mathrm{D}-01$ \\
\hline & & $5.006803270228157 \mathrm{D}+00$ & $9.876285579741800 \mathrm{D}-01$ \\
\hline & & $6.000815466735179 \mathrm{D}+00$ & $9.979929183863017 \mathrm{D}-01$ \\
\hline & & $7.000045035079542 \mathrm{D}+00$ & $9.998470620634641 \mathrm{D}-01$ \\
\hline & & $8.000000738923901 \mathrm{D}+00$ & $9.999962891645340 \mathrm{D}-01$ \\
\hline & & $9.000000000000000 \mathrm{D}+00$ & $9.999999946893169 \mathrm{D}-01$ \\
\hline
\end{tabular}


TABLE 8. The nodes $v_{1}, \ldots, v_{j}$ and weights $u_{1}, \ldots, u_{j}$ for the quadrature rule $S_{n}^{j k a b}(g)=h \sum_{i=1}^{j} u_{i} g\left(v_{i} h\right)+h \sum_{i=0}^{n-1} g(a h+i h)+h \sum_{i=1}^{k} w_{i} g\left(1-x_{i} h\right)$, with $h=(n+a+b-1)^{-1}$, for $g(x)=\phi(x) \log x+\psi(x)$, with $\phi$ and $\psi$ regular functions. The error is of order $O\left(h^{l} \log h\right)$. The nodes $x_{1}, \ldots, x_{k}$ and weights $w_{1}, \ldots, w_{k}$ are found in Table 6.

\begin{tabular}{|c|c|c|c|}
\hline $\bar{l}$ & $a$ & $v_{i}$ & $u_{i}$ \\
\hline 2 & 1 & $1.591549430918953 \mathrm{D}-01$ & $5.000000000000000 \mathrm{D}-01$ \\
\hline \multirow[t]{2}{*}{3} & 2 & $1.150395811972836 \mathrm{D}-01$ & $3.913373788753340 \mathrm{D}-01$ \\
\hline & & $9.365464527949632 \mathrm{D}-01$ & $1.108662621124666 \mathrm{D}+00$ \\
\hline \multirow[t]{3}{*}{4} & 2 & $2.379647284118974 \mathrm{D}-02$ & $8.795942675593887 \mathrm{D}-02$ \\
\hline & & $2.935370741501914 \mathrm{D}-01$ & $4.989017152913699 \mathrm{D}-01$ \\
\hline & & $1.023715124251890 \mathrm{D}+00$ & $9.131388579526912 \mathrm{D}-01$ \\
\hline \multirow[t]{4}{*}{5} & 3 & $2.339013027203800 \mathrm{D}-02$ & $8.609736556158105 \mathrm{D}-02$ \\
\hline & & $2.854764931311984 \mathrm{D}-01$ & 4.8470196854 17959D-01 \\
\hline & & $1.005403327220700 \mathrm{D}+00$ & $9.152988869123725 \mathrm{D}-01$ \\
\hline & & $1.994970303994294 \mathrm{D}+00$ & $1.013901778984250 \mathrm{D}+00$ \\
\hline & 3 & $4.004884194926570 \mathrm{D}-03$ & $1.671879691147102 \mathrm{D}-02$ \\
\hline & & $7.745655373336686 \mathrm{D}-02$ & $1.636958371447360 \mathrm{D}-01$ \\
\hline & & $3.972849993523248 \mathrm{D}-01$ & $4.981856569770637 \mathrm{D}-01$ \\
\hline & & $1.075673352915104 \mathrm{D}+00$ & $8.372266245578912 \mathrm{D}-01$ \\
\hline & & $2.003796927111872 \mathrm{D}+00$ & $9.841730844088381 \mathrm{D}-01$ \\
\hline \multirow{7}{*}{\multicolumn{2}{|c|}{8}} & $6.531815708567918 \mathrm{D}-03$ & $2.462194198995203 \mathrm{D}-02$ \\
\hline & & $9.086744584657729 \mathrm{D}-02$ & $1.701315866854178 \mathrm{D}-01$ \\
\hline & & $3.967966533375878 \mathrm{D}-01$ & $4.609256358650077 \mathrm{D}-01$ \\
\hline & & $1.027856640525646 \mathrm{D}+00$ & $7.947291148621894 \mathrm{D}-01$ \\
\hline & & $1.945288592909266 \mathrm{D}+00$ & $1.008710414337933 \mathrm{D}+00$ \\
\hline & & $2.980147933889640 \mathrm{D}+00$ & $1.036093649726216 \mathrm{D}+00$ \\
\hline & & $3.998861349951123 \mathrm{D}+00$ & $1.004787656533285 \mathrm{D}+00$ \\
\hline \multirow[t]{10}{*}{10} & 6 & $1.175089381227308 \mathrm{D}-03$ & $4.560746882084207 \mathrm{D}-03$ \\
\hline & & $1.877034129831289 \mathrm{D}-02$ & $3.810606322384757 \mathrm{D}-02$ \\
\hline & & $9.686468391426860 \mathrm{D}-02$ & $1.293864997289512 \mathrm{D}-01$ \\
\hline & & $3.004818668002884 \mathrm{D}-01$ & $2.884360381408835 \mathrm{D}-01$ \\
\hline & & $6.901331557173356 \mathrm{D}-01$ & $4.958111914344961 \mathrm{D}-01$ \\
\hline & & $1.293695738083659 \mathrm{D}+00$ & 7.0771546005 94529D-01 \\
\hline & & $2.090187729798780 \mathrm{D}+00$ & $8.741924365285083 \mathrm{D}-01$ \\
\hline & & $3.016719313149212 \mathrm{D}+00$ & $9.661361986515218 \mathrm{D}-01$ \\
\hline & & $4.001369747872486 \mathrm{D}+00$ & $9.957887866078700 \mathrm{D}-01$ \\
\hline & & $5.000025661793423 \mathrm{D}+00$ & $9.998665787423845 \mathrm{D}-01$ \\
\hline
\end{tabular}


TABle 8. (CONTINUED)

\begin{tabular}{|c|c|c|c|}
\hline$l$ & $a$ & $v_{i}$ & $u_{i}$ \\
\hline \multirow[t]{11}{*}{12} & 7 & $1.674223682668368 \mathrm{D}-03$ & $6.364190780720557 \mathrm{D}-03$ \\
\hline & & $2.441110095009738 \mathrm{D}-02$ & $4.723964143287529 \mathrm{D}-02$ \\
\hline & & $1.153851297429517 \mathrm{D}-01$ & $1.450891158385963 \mathrm{D}-01$ \\
\hline & & $3.345898490480388 \mathrm{D}-01$ & $3.021659470785897 \mathrm{D}-01$ \\
\hline & & $7.329740531807683 \mathrm{D}-01$ & $4.984270739715340 \mathrm{D}-01$ \\
\hline & & $1.332305048525433 \mathrm{D}+00$ & $6.971213795176096 \mathrm{D}-01$ \\
\hline & & $2.114358752325948 \mathrm{D}+00$ & $8.577295622757315 \mathrm{D}-01$ \\
\hline & & $3.026084549655318 \mathrm{D}+00$ & $9.544136554351155 \mathrm{D}-01$ \\
\hline & & $4.003166301292590 \mathrm{D}+00$ & $9.919938052776484 \mathrm{D}-01$ \\
\hline & & $5.000141170055870 \mathrm{D}+00$ & $9.994621875822987 \mathrm{D}-01$ \\
\hline & & $6.000001002441859 \mathrm{D}+00$ & $9.999934408092805 \mathrm{D}-01$ \\
\hline \multirow[t]{14}{*}{14} & 9 & $9.305182368545380 \mathrm{D}-04$ & $3.545060644780164 \mathrm{D}-03$ \\
\hline & & $1.373832458434617 \mathrm{D}-02$ & 2.6815140315 76498D-02 \\
\hline & & $6.630752760779359 \mathrm{D}-02$ & $8.504092035093420 \mathrm{D}-02$ \\
\hline & & $1.979971397622003 \mathrm{D}-01$ & $1.854526216643691 \mathrm{D}-01$ \\
\hline & & $4.504313503816532 \mathrm{D}-01$ & $3.251724374883192 \mathrm{D}-01$ \\
\hline & & $8.571888631101634 \mathrm{D}-01$ & $4.911553747260108 \mathrm{D}-01$ \\
\hline & & $1.434505229617112 \mathrm{D}+00$ & $6.622933417369036 \mathrm{D}-01$ \\
\hline & & 778341377 & $40510 \mathrm{D}-01$ \\
\hline & & 3.0479550683863 & $9.235595514944174 \mathrm{D}-01$ \\
\hline & & 4.0049749068134 & $9.821609923744658 \mathrm{D}-01$ \\
\hline & & $4.998525901820967 \mathrm{D}+00$ & $1.000047394596121 \mathrm{D}+00$ \\
\hline & & $5.999523015116678 \mathrm{D}+00$ & $1.000909336693954 \mathrm{D}+00$ \\
\hline & & $6.999963617883990 \mathrm{D}+00$ & $1.000119534283784 \mathrm{D}+00$ \\
\hline & & $7.999999488130134 \mathrm{D}+00$ & $1.000002835746089 \mathrm{D}+00$ \\
\hline \multirow[t]{15}{*}{16} & 10 & $8.371529832014113 \mathrm{D}-04$ & $3.190919086626234 \mathrm{D}-03$ \\
\hline & & 1.2393827255426 & $2.423621380426338 \mathrm{D}-02$ \\
\hline & & 6.009 & $53088 \mathrm{D}-02$ \\
\hline & & 1.8059912496019 & $1.704889420286369 \mathrm{D}-01$ \\
\hline & & $4.142832599028031 \mathrm{D}-01$ & $3.029123478511309 \mathrm{D}-01$ \\
\hline & & $7.964747731112430 \mathrm{D}-01$ & $4.652220834914617 \mathrm{D}-01$ \\
\hline & & $1.348993882467059 \mathrm{D}+00$ & $6.401489637096768 \mathrm{D}-01$ \\
\hline & & $2.073471660264395 \mathrm{D}+00$ & $8.051212946181061 \mathrm{D}-01$ \\
\hline & & $2.947904939031494 \mathrm{D}+00$ & $9.362411945698647 \mathrm{D}-01$ \\
\hline & & $3.928129252248612 \mathrm{D}+00$ & $1.014359775369075 \mathrm{D}+00$ \\
\hline & & $4.957203086563112 \mathrm{D}+00$ & $1.035167721053657 \mathrm{D}+00$ \\
\hline & & $5.986360113977494 \mathrm{D}+00$ & $1.020308624984610 \mathrm{D}+00$ \\
\hline & & $6.997957704791519 \mathrm{D}+00$ & $1.004798397441514 \mathrm{D}+00$ \\
\hline & & $7.999888757524622 \mathrm{D}+00$ & $1.000395017352309 D+00$ \\
\hline & & $8.999998754306120 \mathrm{D}+00$ & $1.000007149422537 \mathrm{D}+00$ \\
\hline
\end{tabular}



